\title{
Accretion and jets in a low luminosity AGN: the nucleus of NGC 1052
}

\author{
S. Falocco ${ }^{1, \star}$, J. Larsson $^{1}$, and S. Nandi ${ }^{2}$ \\ ${ }^{1}$ KTH, Department of Physics, and the Oskar Klein Centre, AlbaNova, SE-106 91 Stockholm, Sweden \\ ${ }^{2}$ National Centre for Radio Astrophysics, TIFR, Pune University Campus, Post Bag 3, Pune 411 007, India \\ Accepted XXX. Received YYY
}

\begin{abstract}
Aims. We aim to determine the properties of the central region of NGC 1052 using X-ray and radio data. NGC 1052 ( $\mathrm{z}=0.005)$ has been investigated for decades in different energy bands and shows radio lobes and a low luminosity active galactic nucleus (LLAGN). Methods. We use X-ray images from Chandra and radio images from Very Large Array (VLA) to explore the morphology of the central area. We also study the spectra of the nucleus and the surrounding region using observations from Chandra and XMM-Newton. Results. We find diffuse soft X-ray radiation and hotspots along the radio lobes. The spectrum of the circum-nuclear region is well described by a thermal plasma $(\mathrm{T} \sim 0.6 \mathrm{keV})$ and a power law with photon index $\Gamma \sim 2.3$. The nucleus shows a hard power law $(\Gamma \sim 1.4)$ modified by complex absorption. A narrow iron $\mathrm{K} \alpha$ line is also clearly detected in all observations, but there is no evidence for relativistic reflection.

Conclusions.

The extended emission is consistent with originating from extended jets and from jet-triggered shocks in the surrounding medium. The hard power-law emission from the nucleus and the lack of relativistic reflection supports the scenario of inefficient accretion in an Advection Dominated Accretion Flow (ADAF).
\end{abstract}

Key words. X-rays: galaxies - Galaxies: nuclei - Galaxies: jets

\section{Introduction}

Low Ionisation Nuclear Emission Line Regions (LINER) are characterised by intense optical lines from low ionisation species (Heckman 1980). Although their optical properties define them as a class, there is not yet a consensus on their ionising mechanism. Indeed, the LINER class is highly heterogeneous, including sources with and without clear evidence of an Active Galactic Nucleus (AGN), as well as both actively star-forming and passive galaxies. Alternatives to photoionisation explain the line excitation as shock-heated regions of the Inter Stellar Medium (ISM) (Dopita et al. 1997; Sugai \& Malkan 2000), strongly star forming regions (Armus et al. 1990; Balmaverde \& Capetti 2015) or diffuse ionised plasmas (Collins \& Rand 2001). However, several LINER surveys have shown that the majority of sources are AGN powered by low-luminosity accretion onto SMBHs (Ho 2008; Terashima et al. 2002; González-Martín et al. 2009b; Hernández-García et al. 2013; Hernández-García et al. 2014). The evidence collected so far provides support for the scenario that these sources have ADAFs embedded in standard accretion discs, as described by Narayan et al. (1998). This picture has been adopted to explain most LINER as LLAGN (Ho 2008). Since low and high ionisation states correspond to low and high accretion rates, LINER can be seen as the low accretion rate extension of Seyfert galaxies (Alexander \& Hickox 2012). LINER thus offer an opportunity to study SMBHs in the low accretion rate regime, which is thought to dominate in the local universe (e.g. Hickox et al. 2009; Alonso-Herrero et al. 2008).

\footnotetext{
^ E-mail: falocco@kth.se
}

The X-ray emission from LINER offers a powerful way of unveiling the presence of an AGN. Indeed, both spectral features and variability support the scenario of SMBH accretion in LINER (González-Martín et al. 2009a; Hernández-García et al. 2014; Hernández-García et al. 2016). One unambiguous evidence of AGN-like accretion is the narrow iron $\mathrm{K} \alpha$ line at $6.4 \mathrm{keV}$. This line is ubiquitous in AGN and originates from the outer parts of the accretion disk or regions further away, such as the torus or Broad Line Region. AGN commonly also exhibit a relativistically broadened iron line emitted from the innermost accretion disc, along with other reflection features such as the Compton hump above $10 \mathrm{keV}$ (e.g., García et al. 2014). Such relativistic reflection is not expected to be observed in the ADAF scenario for LINER, as the inner accretion flow is then optically thin and the iron is fully ionised. A relativistic line has been reported in only one source (NGC 1052, Brenneman et al. 2009), discussed further below. The narrow iron line is instead rather common (e.g. $42 \%$ in the sample of Terashima et al. 2002), offering evidence for the presence of AGN.

The X-ray spectra of LINER often have significant contributions from a variety of components in addition to the emission associated with accretion. Such components can be detected due to the typical low redshifts and intrinsic weakness of the nuclei. In soft X-rays, a mixture of radiation from supernova remnants and starburst regions are sometimes used to model the thermal X-ray continuum (González-Martín et al. 2009a). Emission from supernovae and hot ( 10 keV) gas from starburst activity can also contribute at hard X-rays (Terashima et al. 2002). Some LINER also exhibit X-ray emission from jets (Hardcastle \& Worrall 1999), which can be be due to synchrotron emission or inverse Compton scattering 
(e.g. Worrall 2009; Hardcastle \& Worrall 1999, Tavecchio et al. 2000; Celotti et al. 2001)

NGC 1052 is considered a LINER prototype. It has been studied extensively in the literature (e.g. Heckman 1980 and Kadler et al. 2004a) and been included in numerous X-ray surveys of LINER (González-Martín et al. 2009b; Hernández-García et al. 2013; Hernández-García et al. 2014; González-Martín et al. 2014, 2015; Hernández-García et al. 2016). It is hosted by an elliptical galaxy located at a distance of 19.2 Mpc (Tully et al. 2013) with redshift $z=0.005$ (Jensen et al. 2003), and its black hole mass has been estimated to $1.5 \times 10^{8} \mathrm{M}_{\odot}$ (Woo \& Urry 2002). It exhibits kpc-scale twin radio jets and associated lobes (e.g., Kadler et al. 2004a). On pcscales the two jets are separated by a central gap that becomes smaller with increasing frequencies, which has been interpreted as absorption from a torus surrounding the nucleus (Kadler et al. 2004b; Baczko et al. 2019). There is also optical line emission aligned with the inner radio jet, presumably originating from shock-excited regions (Kadler et al. 2004a; Dopita et al. 2015).

NGC 1052 was first resolved in X-rays in a short Chandra observation from 2001, which revealed soft X-ray emission associated with the kpc-scale radio jets (Kadler et al. 2004a). This extended emission was well described by a $0.4-0.5 \mathrm{keV}$ thermal plasma and interpreted as originating from jet-triggered shocks. The broad-band X-ray emission from NGC 1052 is, however, dominated by the nucleus. Early studies of the X-ray spectrum revealed a flat power-law continuum affected by significant intrinsic absorption, as well as a narrow Fe line at $6.4 \mathrm{keV}$ (Guainazzi \& Antonelli 1999; Guainazzi et al. 2000). The flat spectrum was interpreted as Bremsstrahlung from an ADAF. A more detailed study based on a Suzaku observation from 2007 confirmed the basic picture of an absorbed hard power-law, but also revealed the presence of a relativistically broadened $\mathrm{Fe}$ line (Brenneman et al. 2009). However, no associated Compton reflection was detected, making an origin in the inner region of a standard accretion disk unlikely. Brenneman et al. (2009) instead suggested that the broad line may have been produced near the base of the jet.

More recently, Osorio-Clavijo et al. (2020) presented an extensive study of NGC 1052 using observations from Chandra, XMM-Newton, Nustar, and Suzaku. Their preferred model for the nucleus comprises an absorbed power law and reflection from distant material, while the spectrum of the circumnuclear region was fitted with a very hard power law together with a thermal component, consistent with Kadler et al. (2004a). Osorio-Clavijo et al. (2020) also reported variability in the nucleus.

From the current knowledge and previous work on NGC 1052, it is clear that open questions still remain. One of these concerns the nature and origin of the complex soft X-ray emission from the circum-nuclear region, including the possible role of star formation and the connection with the radio jets. The details of the circum-nuclear spectrum also affects the modelling of the nucleus, including the properties of the complex absorption, spectral variability, and the possible presence of relativistic reflection. The latter point is especially interesting because it is not expected in an ADAF and because there have been discordant results regarding the presence of a broad iron line in the literature.

This paper presents an analysis of one Chandra and four $X M M$-Newton observations of NGC 1052 with the purpose to explore these aspects in more detail. The excellent spatial resolution of Chandra allows us to study the morphology of the central region, as well as the X-ray spectra associated with the jets, the galaxy, and the nucleus. We also present the first comparison between the long Chandra observation of 2005 and the VLA observations. The information obtained from Chandra is also used when analysing the XMM-Newton observations, which offer good-quality spectra but do not resolve the galaxy. This approach allows us to characterise the spectra of the nucleus in detail and to determine whether the variability is significant on time scales of years. We describe the observations in Section 2, present the analysis of images and spectra in Section 3, and discuss the results in Section 4. The conclusions are summarised in Section 5.

\section{Observations and data reduction}

The Chandra and XMM-Newton observations analysed in this work are listed in Table 1 For comparison with the Chandra images, we also retrieved a radio image at $1.4 \mathrm{GHz}$ from the VLA archive.

\subsection{Chandra observation}

There are two Chandra observations of NGC 1052, one from 2001 (observation Identification, OBSID 385) and one from 2005 (OBSID 5910). Both observations were performed with the ACIS-S instrument. We analysed only the 2005 observation because it has a significantly longer exposure time and because the first observation has already been presented in Kadler et al. (2004a). The details of the 2005 observation are reported in Table 1 We note that contamination on the optical blocking filter (which affects Chandra data below $\sim 1 \mathrm{keV}$ ) was small at the time of the observation and hence does not significantly affect our analysis (Plucinsky et al. 2018).

The data were reduced using CIAO v4.9 (Fruscione et al. 2006), with calibration files version 4.7.4. We checked that there was no background flaring present in the observation. However, the nucleus is affected by pileup at a level of $\sim 10$ per cent (estimated using PIMMS 1 ). To account for this potential problem we extracted the spectrum of the nucleus from an annular region with inner and outer radii of $1^{\prime \prime}$ and $6^{\prime \prime}$, respectively. The background was extracted in a source-free nearby circular region with a radius of $10^{\prime \prime}$. The spectrum was binned to 25 counts per bin in order to allow for fitting using $\chi^{2}$ statistics.

We also extracted spectra in two rectangular regions located to the east and west of the nucleus in order to analyse the emission from the jet and galaxy (see Fig. 1). The eastern box is centred at 2:41:05.501, -8:15:21.65 (equatorial coordinates in J2000.0 epoch) with a size of $12.34 \times 8.07^{\prime \prime}{ }^{2}$, while the western box is centred at 2:41:04.319, $-8: 15: 21: 30$ with a size of $7.12 \times 8.30^{\prime \prime} 2$. We underline that these areas exclude the central region where the nuclear emission dominates (see Fig. 1). Given the low number of counts in these regions (346 and 262 counts in the east and west, respectively), we binned the spectra to have one count per bin and used Cash statistics for the analysis. The spectra were fitted over the $0.5-7 \mathrm{keV}$ energy range as there was no detection above $7 \mathrm{keV}$. We also extracted spectra in regions located to the north and south of the nucleus, but found that the spectra did not have sufficient signal for analysis.

\subsection{XMM-Newton observations}

The details of the four XMM-Newton observations of NGC 1052 analysed in this work are summarised in Table 1 There is also

http://heasarc.gsfc.nasa.gov/docs/software/tools/pimms.html 
Table 1: Details of the X-ray observations. Exp is the exposure time while exp clean $_{\text {in }}$ the net exposure remaining after removing times affected by background flares from the instruments specified (MOS1+MOS2 or pn). Count rates are given for the $0.5-10 \mathrm{keV}$ energy range for the different instruments (MOS1+MOS2, pn and ACIS). Superscript 1 means that only MOS1 was used. In the case of Chandra ACIS, the count rate is for the annular extraction region at the nucleus.

\begin{tabular}{|c|c|c|c|c|c|c|c|c|}
\hline Date & Telescope & OBSID & $\begin{array}{l}\exp \\
{[\mathrm{ks}]}\end{array}$ & $\begin{array}{c}\text { MOS } \\
\text { exp clean } \\
{[\mathrm{ks}]}\end{array}$ & $\begin{array}{c}\mathrm{pn} \\
\exp _{\text {clean }} \\
{[\mathrm{ks}]}\end{array}$ & $\begin{array}{c}\text { MOS } \\
\text { rate } \\
{\left[\operatorname{cts~s}^{-1}\right]}\end{array}$ & $\begin{array}{c}\text { pn } \\
\text { rate } \\
{\left[\mathrm{cts} \mathrm{s}^{-1}\right]}\end{array}$ & $\begin{array}{c}\text { ACIS } \\
\text { rate } \\
{\left[\operatorname{cts~s}^{-1}\right]}\end{array}$ \\
\hline 2001 Aug 15 & XMM-Newton & 0093630101 & 16.3 & - & 10.4 & - & 0.4 & - \\
\hline 2005 Sept 18 & Chandra & 5910 & 59.2 & - & - & - & - & 0.037 \\
\hline 2006 Jan 12 & XMM-Newton & 0306230101 & 54.9 & 100.0 & 39.8 & 0.16 & 0.5 & - \\
\hline 2009 Jan 14 & XMM-Newton & 0553300301 & 52.3 & $47.0^{1}$ & 39.9 & $0.2^{1}$ & 0.6 & - \\
\hline 2009 Aug 12 & XMM-Newton & 0553300401 & 59.0 & 110.0 & 41.8 & 0.18 & 0.6 & - \\
\hline
\end{tabular}

a more recent XMM-Newton observation available (from 2017 January 17), but we did not analyse this since the source lies very close to a chip gap in the pn and overlaps with a bright column in MOS2. The four observations analysed are separated by seven months to five years, which is suitable for investigating possible spectral variability of the nucleus. From these observations we have used all data except those where the source falls on a chip gap, which turns out to be the case for the first MOS observation in 2001 and the MOS2 observation from 2009 January 14. All the observations were performed in full frame mode with the medium filter.

For the data reduction we processed the observation data files using SAS v15.0.0 and the calibration files XMM-CCF-REL349 , following standard procedures 2 All the observations were moderately affected by time periods with strong background flares, which we excluded from the analysis. These time periods correspond to $\sim 30$ per cent of the total exposures (see Table 11. We also verified that the observations were not affected by pileup using the tool EPATPLOT. Source spectra were extracted from circular regions with radii of 55" (following Evans et al. 2007 for bright sources observed with Swift, which has an angular resolution similar to XMM-Newton). The only exception to this was when the source fell close to a chip gap, in which case we reduced the extraction region to $30^{\prime \prime}$. The background spectra were extracted from circular regions with $\sim 80^{\prime \prime}$ radii located near the source on the same detector chip.

The spectra from the MOS1 and MOS2 instruments were merged with a similar procedure as in Falocco et al. (2014). The merging procedure computes the sum of the exposures and the counts, while the backscale is the exposure-weighted average of the backscales of the individual spectra. The RMFs and ARFs were similarly combined using the ftools task ADDRMF. The resulting spectra were binned in order to have at least 25 counts per bin and to not over-sample the spectral resolution by more than a factor three. The spectral analysis was performed over the $0.5-10 \mathrm{keV}$ energy range using $\chi^{2}$ statistics.

\subsection{Radio data}

We used radio observations from the Very Large Array (VLA) performed on 2002 January 26 (proposal code AC0622). The data consist of a short observation with a total exposure time of 5 min on the source. The observations were performed in A configuration in the $\mathrm{L}$ band (central observing frequency $1.4 \mathrm{GHz}$ and bandwidth $50 \mathrm{MHz}$ ). The beam size is $1.79 \times 1.33$ ".

\footnotetext{
2 'Users Guide to the XMM-Newton Science Analysis System', Issue 15.0, 2019 (ESA: XMM-Newton SOC)
}

\section{Data analysis and results}

We start the analysis by investigating the morphology of the galaxy and jet using Chandra and VLA images in Section 3.1 We then present the X-ray spectral analysis in Section 3.2, where we first characterise the Chandra spectrum of the extended emission and then analyse the XMM-Newton and Chandra spectra of the nucleus. We focus our analysis on time-averaged spectra, which allow us to investigate possible long-term variations. The light curves of the individual XMM-Newton observations (except the first short one) have already been studied by Hernández-García et al. (2014), who showed that no significant variability was present during the observations.

\subsection{Imaging}

We produced exposure-corrected images from Chandra using the FLUXIMAGE tool in CIAO, using monochromatic energies to calculate the exposure maps. The images were extracted in different energy bands in order to probe a variety of different emission components, including the AGN-like nucleus, the jet, as well as the galaxy, where X-ray emission may arise from the ISM, star forming regions, binaries etc. We used the energy bands $0.5-1,1-2,2-6$ and $6-10 \mathrm{keV}$, with nominal energies of $0.9,1.5,3.8$ and $8.0 \mathrm{keV}$, respectively. The images are plotted in Fig. 11 where the two highest energy bands are plotted together in the right panel. Comparing the images, we note that the emission becomes less extended as the energy increases, with the radiation from the nucleus dominating at the highest energies. At energies below $1 \mathrm{keV}$, we see diffuse emission from the galaxy over an extended scale. Between 1 and $2 \mathrm{keV}$, there is less extended diffuse emission as well as a small number of discrete sources. Only the nucleus is clearly detected in the images above $2 \mathrm{keV}$.

The X-ray emission at low energies extends $\sim 10^{\prime \prime}$ from the nucleus, with most of the emission being concentrated along the east-west direction. The X-ray emission is more compact than the optical galaxy, which has a diameter of 100" according to NED 3 . However, the X-ray emission is similar in scale to the radio jets. This is seen in Fig. 2, where we compare the VLA $1.4 \mathrm{GHz}$ observation from 2002 with the Chandra images in the $0.5-1$ and $1-2 \mathrm{keV}$ energy ranges. The VLA image shows large scale radio structure which is extended over $2.82 \mathrm{kpc}$. The radio jet has also been studied on pc-scales using VLBI observations (e.g., Kadler et al. 2004b; Baczko et al. 2016, 2019), but these small scales are not resolved with Chandra.

\footnotetext{
3 The NASA/IPAC Extragalactic Database (NED) is operated by the Jet Propulsion Laboratory, California Institute of Technology, under contract with the National Aeronautics and Space Administration
} 

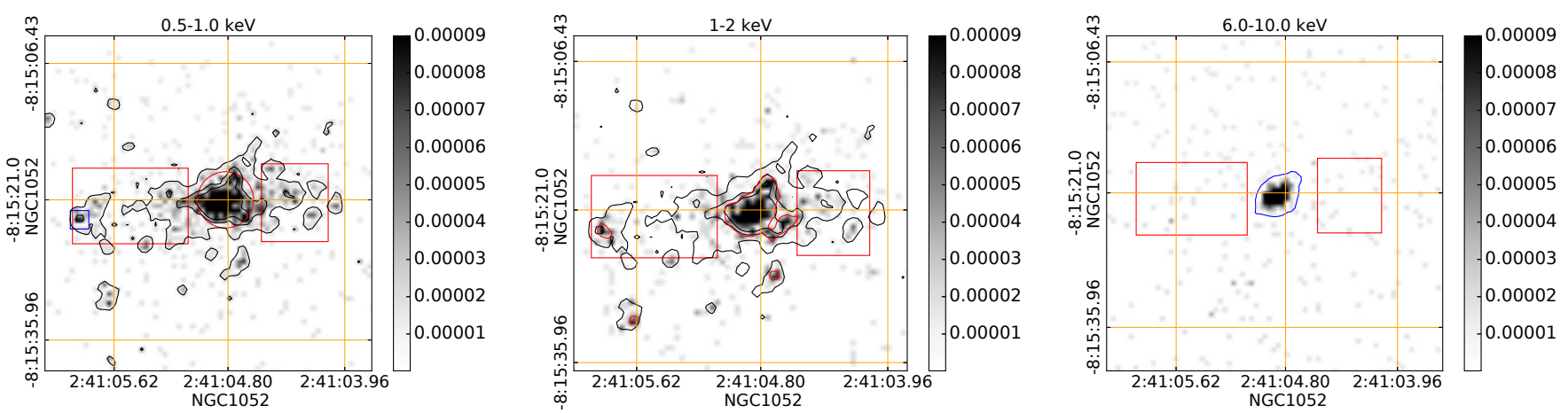

Fig. 1: Chandra images of NGC 1052 (units of $\left.\mathrm{cts} \mathrm{s}^{-1}\right)$ covering the central $22^{\prime \prime} \times 27^{\prime \prime}$ region $(\sim 2.4 \times 2.9 \mathrm{kpc})$. The orange grid shows units of $11.1^{\prime \prime} \times 13.3^{\prime \prime}$, where $11.1^{\prime \prime}$ corresponds to $1.2 \mathrm{kpc}$ at $\mathrm{z}=0.005$. Left: Image in the $0.5-1 \mathrm{keV}$ energy range together with black contours corresponding to flux levels at 5, 20 and $35 \sigma$ above the background. The red circle shows the outer radius of the spectral extraction region for the nucleus and the red rectangles show the spectral extraction regions used for the extended emission from the jet and galaxy. A hotspot associated with the eastern radio lobe is highlighted by a blue rectangle (the radio lobe can be seen in Fig. 2). Centre: image in the $1-2 \mathrm{keV}$ energy range together with a red contour corresponding to $5 \sigma$. The contours for the $0.5-1 \mathrm{keV}$ image in the left panel are also shown for reference. Right: Image in the $6-10 \mathrm{keV}$ energy band. The blue contour shows the $5 \sigma$ level from the $2-6 \mathrm{keV}$ image.
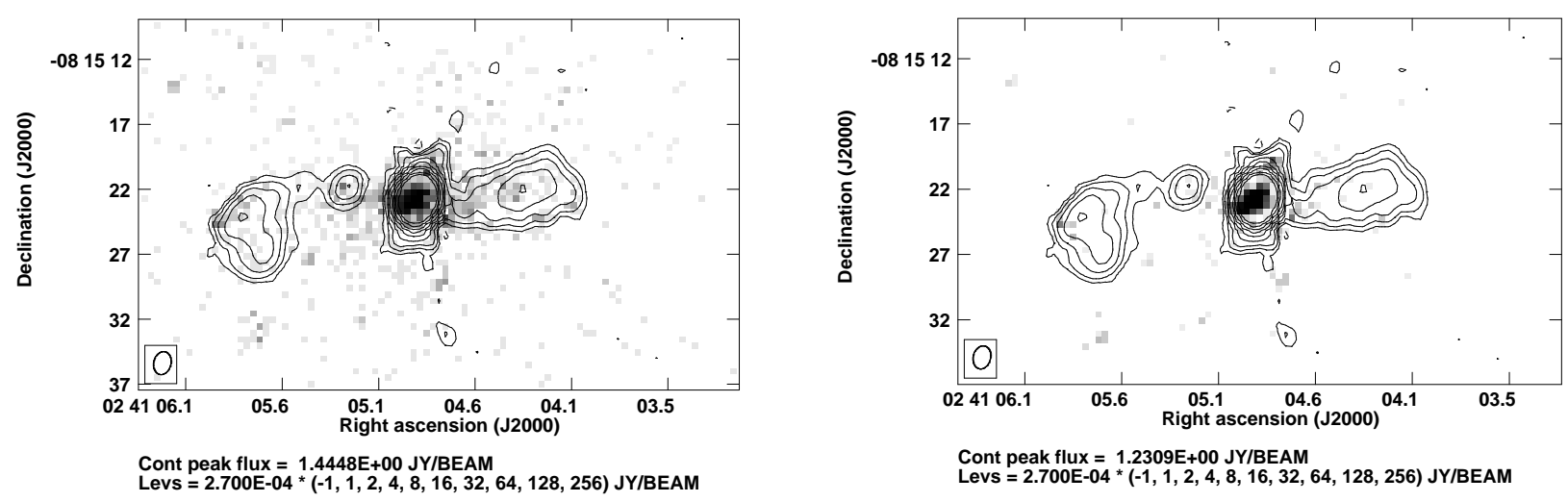

Fig. 2: Comparison of Chandra and VLA images of NGC 1052. The left and right panels show the Chandra images over $0.5-1$ and $1-2 \mathrm{keV}$, respectively (see also Fig. 1). The superposed contours are from the VLA $1.4 \mathrm{GHz}$ observation from 2002. Contour levels are $3 \sigma(-1,1,2,4,8 \ldots .256)$, where $\sigma=0.09 \mathrm{mJy} /$ beam the image root mean square.

The VLA image at $1.4 \mathrm{GHz}$ shows two compact high surface brightness regions, or two hotspots, on the eastern lobe. These have also been seen in a MERLIN observation from 1995 (Kadler et al. 2004a, labelled as H1 and A in their Fig. 3). On the western lobe, one main hotspot is seen, with some indication of an inner secondary one. The latter was seen more clearly in the MERLIN observation (Kadler et al. 2004a). In the X-ray images, a hotspot below $2 \mathrm{keV}$ is seen along the outer edge of the eastern radio lobe. This X-ray emission is likely due to shocks in the region where the extended jet interacts with the environment, as discussed in (Kadler et al. 2004a). On the same lobe, there is also some X-ray emission below $1 \mathrm{keV}$ at the position of the inner radio hotspot. However, it is not clear if this is associated with the jet since X-ray emission at a similar level is also seen in the nearby regions that do not overlap with the radio contours. The western radio lobe has a less clear counterpart in the $\mathrm{X}$-ray band, but we note some low surface brightness emission coincident with the southern boundary of the lobe.

\subsection{Spectral analysis}

We performed the spectral analysis using XSPEC version 12.9. All fits described below include Galactic absorption modelled with tbabs. The Galactic $\mathrm{H}$ column density in the direction of NGC 1052 is $3.1 \times 10^{20} \mathrm{~cm}^{-2}$, determined using the NHTOT tool 4, which takes into account the contribution from molecular hydrogen. We first analyse the Chandra spectra of the extended jet plus galaxy regions, in order to use the best-fitting model for the analysis of the XMM-Newton spectra, where the galaxy is unresolved.

The XMM-Newton EPIC pn and MOS spectra were fitted simultaneously, with all parameters tied except for a crossnormalisation factor. We finally disuss the Chandra spectrum of the nucleus. This has relatively low signal-to-noise, and the analysis is therefore guided by the results from the XMM-Newton spectra. We quote uncertainties on fit parameters at 90 per cent significance for one interesting parameter $\left(\Delta \chi^{2}=2.7\right)$.

\footnotetext{
$\overline{4}$ http://www.swift.ac.uk/analysis/nhtot/docs.php
} 
Table 2: Results from fits to the Chandra spectra of the extended emission from the jet and galaxy. The fluxes are unabsorbed.

\begin{tabular}{|c|c|}
\hline Parameter & Value \\
\hline \multicolumn{2}{|l|}{ tbabs*mekal } \\
\hline$T[\mathrm{keV}]$ & $0.79_{-0.04}^{+0.03}$ \\
\hline cstat/d.o.f. & $477.8 / 248$ \\
\hline \multicolumn{2}{|l|}{ tbabs*(mekal+mekal) } \\
\hline$T_{\mathrm{h}}[\mathrm{keV}]$ & $5.3_{-1.9}^{+5.7}$ \\
\hline$T_{\mathrm{c}}[\mathrm{keV}]$ & $0.32_{-0.03}^{+0.03}$ \\
\hline cstat/d.o.f. & $269.8 / 246$ \\
\hline \multicolumn{2}{|l|}{ tbabs*pow } \\
\hline$\Gamma$ & $2.7_{-0.1}^{+0.1}$ \\
\hline cstat/d.o.f. & $292.4 / 248$ \\
\hline \multicolumn{2}{|l|}{ tbabs*(pow+mekal) } \\
\hline$\Gamma$ & $2.3_{-0.1}^{+0.2}$ \\
\hline$T[\mathrm{keV}]$ & $0.6_{-0.1}^{+0.1}$ \\
\hline cstat/d.o.f. & $258.4 / 246$ \\
\hline$F_{\text {tot,0.5-7 }}\left[\mathrm{erg} \mathrm{cm}^{-2} \mathrm{~s}^{-1}\right]$ & $6.8 \pm 1.1 \times 10^{-14}$ \\
\hline$L_{\mathrm{tot}, 0.5-7}\left[\mathrm{erg} \mathrm{s}^{-1}\right]$ & $3.5 \pm 0.6 \times 10^{39}$ \\
\hline$L_{\mathrm{th}, 0.5-7}\left[\mathrm{erg} \mathrm{s}^{-1}\right]$ & $8.6 \pm 2.6 \times 10^{38}$ \\
\hline$F_{\mathrm{tot}, 0.5-2}\left[\mathrm{erg} \mathrm{s}^{-1} \mathrm{~cm}^{-2}\right]$ & $4.9 \pm 0.9 \times 10^{-14}$ \\
\hline$L_{\mathrm{tot}, 0.5-2}\left[\mathrm{erg} \mathrm{s}^{-1}\right]$ & $2.4 \pm 0.5 \times 10^{39}$ \\
\hline
\end{tabular}

\subsubsection{Chandra spectra of the extended emission}

The spectra of the extended emission extracted from the two rectangular regions in Fig. 1 show very similar features. We therefore fitted them together with all parameters tied except for a cross nomalisation constant. Due to the limited statistics of the spectra we only considered simple models in the form of a power law (expected from jet emission) and a thermal mekal component. The latter may arise from the ISM, shocks or starburst regions. The results from the fits are reported in Table 2 We found that a single component could not describe the spectra, whereas a pow+mekal and mekal+mekal provided acceptable fits. We adopt the former as our preferred model due to slightly better cstat and the high temperature of the second mekal component in the latter model, which lacks a clear physical interpretation. We also tested adding intrinsic absorption to the models, but found that it was not required. The spectra and best-fitting model are shown in Fig. 3. We refer to our preferred model for the jet plus galaxy region as JGAL hereafter.

\subsubsection{XMM-Newton spectra}

The XMM-Newton spectra of NGC 1052 include emission from the nucleus, extended jet and surrounding galaxy. To account for the latter components we included the Chandra JGAL model described above in all fits to XMM-Newton spectra. We kept all parameters of JGAL fixed at their best-fit values (from Sect. 3.2.1) except for the normalisation (but keeping the same relative normalisation between the two components as in Chandra).

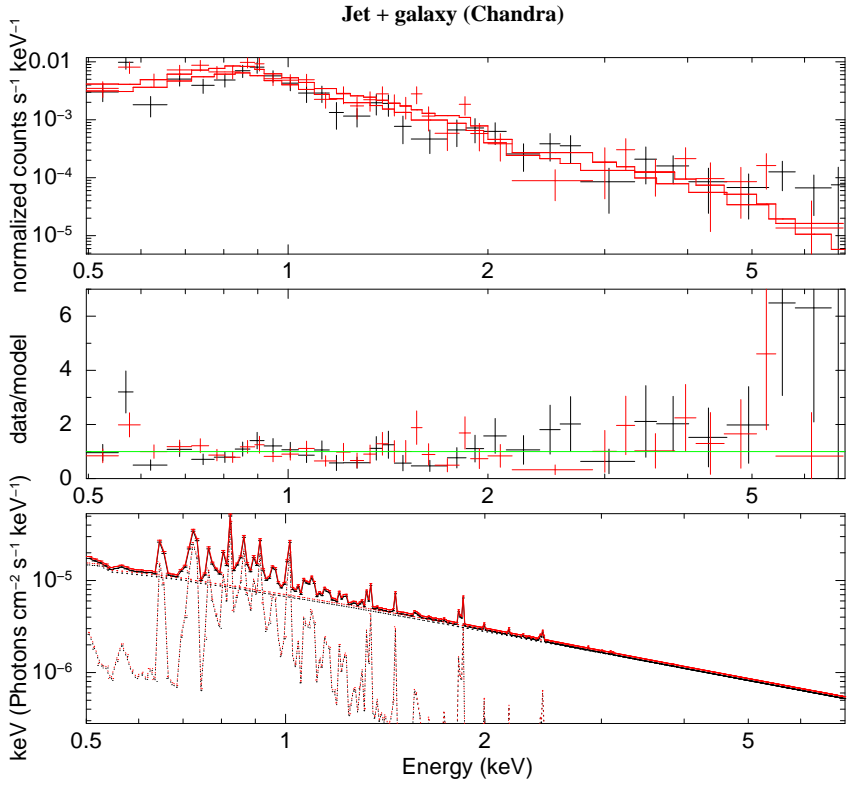

Fig. 3: Chandra spectra of the extended emission from the galaxy plus jet fitted with the pow+ mekal model. The red and black spectra were extracted from the regions east and west of the nucleus, respectively (see Fig. 1). The spectra have been binned for visual purposes only (to have $4 \sigma$ significance in each bin). Top panel: spectra with the best-fit model, central panel: ratio of data/model, bottom panel: model components.

We started by modelling the emission from the nucleus as a partially absorbed power law and a narrow Fe line at $6.4 \mathrm{keV}$, which is the simplest possible model motivated by previous studies of the source (Guainazzi \& Antonelli 1999; Guainazzi et al. 2000; Brenneman et al. 2009). The absorption is assumed to be caused by the molecular torus (e.g., Baczko et al. 2019). In XSPEC the model is tbabs* (zpcfabs*pow+zgauss+mekal+pow). We will refer to it as PG-JGAL from here on. The fit results are reported in Table A.1 in the Appendix. The fitting procedure starts by characterising the continuum between $0.5-5.5$ and $7-10 \mathrm{keV}$ before adding the Gaussian line and fitting the whole energy interval. When adding the line, its energy was fixed at $6.4 \mathrm{keV}$ in order to represent the $\mathrm{K} \alpha$ line from neutral iron. Its width was fixed at $0.09 \mathrm{keV}$ to be intrinsically narrow and broadened only by the spectral resolution of the instruments. Allowing the energy and width of the line to vary did not significantly change the results, confirming a narrow line from neutral iron. The $\chi^{2}$ values obtained when fitting the model without the line are reported in the first row of Table A.1 (where the model without the line is referred to as P-JGAL). A comparison with the fit statistic for the PG-JGAL model in the same table shows that the iron line is highly significant, improving the $\chi^{2}$ by $\sim 100-140$ for one degree of freedom (d.o.f) for the three observations with the longest exposures.

Since the iron line is highly significant, we introduced a complete model which considers Compton reflection and fluorescence in a self consistent way, considering that Compton reflection is expected together with fluorescence. In line with this, Osorio-Clavijo et al. (2020) found evidence for Compton reflection in Nustar and Suzaku observations of the source. To account for this reflection, we substituted the gaussian line in our model with a pexmon component (Nandra et al. 2007). We refer to this model as PexJGAL. Pexmon includes fluorescent 
lines of $\mathrm{Fe} \mathrm{K} \alpha, \mathrm{FeK} \beta$, and $\mathrm{Ni} \mathrm{K} \alpha$, the $\mathrm{Fe} \mathrm{K} \alpha$ Compton shoulder, as well as Compton reflection. We allowed only the normalisation of the pexmon component to vary in the fits. The inclination of the source is known to be high (Baczko et al. 2019) and we fixed it at $85^{\circ}$, noting that its exact value does not affect the conclusions. We further tied the photon index to the value of the primary power law and kept the cutoff energy and abundances fixed at their default values. This model does not produce a satisfactory $\chi^{2}$ (see Table 3) and leaves residuals especially in the soft band below $3 \mathrm{keV}$. For this reason we included an additional absorption component, as also done in the previous Suzaku observation (Brenneman et al. 2009). The overall model in xspec was: tbabs (zpcfabs*absori *pow + pexmon + mekal + pow), hereafter referred to as PexiJGAL.

The model PexiJGAL produces acceptable fits to all spectra, as can be seen in Table 3 . The reflection fraction 5 ranges between 3 and $6 \%$. Fig. 4 shows the spectra fitted with this model, along with the individual model components, and Table 3 reports the fits parameters. The value of the ionisation parameter of the absori component is consistent with null ionisation $\left(\xi<2 \mathrm{erg} \mathrm{cm} \mathrm{s}^{-1}\right.$, see Table 3), showing that the second absorber is consistent with being neutral. Indeed, replacing absori with a cold absorption model (pcfabs with 100 per cent covering fraction) does not significantly change the fit statistic or the other model parameters. Independently of the ionisation degree, the second absorber significantly improves the fit for all $X M M$ Newton spectra, resulting in $\Delta \chi^{2}$ of $>40$ for 2 d.o.f., (Table 3 ). We discuss these results in Sect. 4.3.

Although the PexiJGAL model works well for all spectra, there are still some discrepancies between the observed spectra and the model in the soft band. This is particularly evident in the XMM-Newton spectra of 2006 and of 2009-08-15. We thus tested some modifications to the PexiJGAL model applied to the spectrum of 2009-08-15, which has the best spectral quality. As a first test we allowed the abundances of the mekal component in the JGAL model to vary. We found that the abundances were poorly constrained and that the improvement in the fit was not significant. We then performed a test leaving the abundances of the JGAL model fixed as before, but adding an extra mekal component. We found that a collisional plasma at a temperature $1.3 \pm 0.2 \mathrm{keV}$ significantly improves the fit $\left(\Delta \chi^{2}=32\right.$ for 2 d.o.f $)$. There was no significant change in the other model parameters, showing that the continuum characterisation with PexiJGAL is robust. The additional plasma contributes at most $9 \%$ to the total flux (unabsorbed flux from the AGN plus JGAL component) between 0.5 and $2 \mathrm{keV}$, which is smaller than uncertainties on the total flux itself ( $\sim 13 \%$ in this band). Considering that the test was performed in the best observation, we conclude that the addition of this component would only have a minor effect in the fits to the other spectra.

\section{Constraints on relativistic reflection}

We have tested relativistic reflection components in addition to the PexiJGAL model in order to investigate if any reflection features from the inner accretion disk close to the SMBH are present in the XMM-Newton spectra. We performed the tests only with the first and third observations (OBSIDs 0093630101 and 0553300301). This is because the first spectrum shows an indication of an excess on the red side of the narrow line (see Fig. 4 , panel a), while the third one has a good spectral quality (see Fig. 4 panel c).

\footnotetext{
5 The reflection fraction is derived as the ratio between the fluxes of the reflected radiation to the total AGN radiation between 2 and $10 \mathrm{keV}$
}

We started by adding only a laor model, which represents the line emitted from a disk around a Kerr black hole (Laor 1991). We found that this model did not improve the fit and that the free parameters could not be constrained, showing that no prominent broad line is present. To still obtain some constraints on the line we proceeded by fixing most of the parameters, using information obtained from other studies of the source. Radio observations have shown that the inclination, $i$, is high (Baczko et al. 2016, 2019), although Baczko et al. (2019) finds that there is no value of $i$ that can explain all observations, suggesting that the jets are asymmetric. We therefore performed the fits with $i$ fixed at different values in the range $65^{\circ} \leq i \leq 90^{\circ}$, finding that it did not affect the conclusions. We initially also fixed the inner radius of the disk at $r_{\text {in }}=26 r_{\mathrm{g}} 6$ which is a lower limit estimated by comparing the accretion power of the disk with the spectral energy distribution from sub-arcsec resolution observations (Reb et al. 2018). Finally, we fixed the the outer radius of the disk $\left(r_{\text {out }}\right)$ and the emissivity index $(q)$ at their default values of $r_{\text {out }}=400 r_{\mathrm{g}}$ and $q=3$. This leaves the normalisation as the only free parameter. This model did not significantly improve the fit, resulting in a very similar $\chi^{2}$ and an equivalent width (EW) of the line consistent with zero. Leaving the inner radius free to vary did not change the results, as well as fixing it to its lower value $1.24 r_{g}$.

We also performed fits where we replaced laor with the relxill model (Dauser et al. 2014), which derives the full reflection spectrum from the disc, including line emission and Compton scattering. We fixed $i, q, r_{\text {in }}$ and $r_{\text {out }}$ at the same values as for the laor model. In addition, the photon index of the primary radiation was tied to the value of the power-law model, the ionisation was set to zero, the iron abundance to 1 (in units of solar abundances) and the high-energy cutoff of the primary spectrum was fixed to $300 \mathrm{keV}$. Only the normalisation was thus left free to vary in relxill. This test produced a marginal improvement of the $\chi^{2}\left(\Delta \chi^{2}\right.$ of 3 in the first observation and 2 in the third one for 1 d.o.f. less than the fit with PexiJGAL). Leaving $r_{\text {in }}$ free did not result in any significant improvements. Its best fit value is $14 r_{\mathrm{g}}$ in both observations with upper limits of 370 and $400 r_{\mathrm{g}}$ in the first and third observation, respectively. Since the model PexiJGAL includes Compton reflection from distant material, we have also tested for the presence of a relativistic line after excluding the pexmon component. This allows us to investigate if part of the broad line had been modelled as Compton reflection. We thus added the laor model (with the same constraints as above) to the model referred to as PGI-JGAL in Table A.1 in the Appendix. We found that the inner radius is constrained to $<100 r_{\mathrm{g}}$ in the first observation and to $<90 r_{\mathrm{g}}$ in the third one, with a $\chi^{2}$ similar to the fit without the relativistic line. This confirms that the relativistic line is not significantly required by the data.

\subsubsection{Chandra spectrum of the nucleus}

We finally analysed the Chandra spectrum of the nucleus. This spectrum has significantly lower signal than the XMM-Newton spectra since the brightest part of the PSF was excluded in order to avoid pileup. The Chandra spectrum also differs from the XMM-Newton spectra in that the contribution from the jet+galaxy is expected to be much smaller due to the superior spatial resolution. The results from the fits to the Chandra spectrum are summarised in Table 4 We started by fitting a model composed of an absorbed power law and a Gaussian, equivalent

\footnotetext{
${ }^{6} r_{\mathrm{g}}$ is the gravitational radius $=\frac{G M}{c^{2}}$
} 
to our initial model for the XMM-Newton spectra without the jet plus galaxy model.

Since we found that the narrow iron line is significant, we substituted the gaussian with the self-consistent pexmon model as was done for the XMM-Newton spectra. Since there were residuals in the soft X-rays, we added a thermal model to represent a contribution from the galaxy. The xspec model was tbabs (zpcfabs*powerlaw + pexmon + mekal), hereafter called PexTH. The fit results are presented in Table 4. To take into account some remaining residuals in the soft $\mathrm{X}$ rays and to use a model consistent with the one used for $X M M-N e w t o n$, we finally added an ionised absorber. The xspec model was tbabs (zpcfabs*absori powerlaw + pexmon + mekal), hereafter called PexiTH. The fits results are reported in Table 4 and the spectrum with the best fitting model is shown in Fig. 5.

The primary radiation of the Chandra spectrum of the nucleus is rather flat compared to typical values observed for standard AGN. However, it is consistent with the $\Gamma$ of the second $X M M-N e w t o n$ spectrum, which is the closest in time (observed four months later) within the uncertainties. The column densities of the low and high density absorbers are poorly constrained, with best-fit values similar to the first XMM-Newton observation (observed four years earlier), but higher than the other observations with better spectral quality. The flux of the AGN component in Table 4 has been corrected for the fact that the Chandra extraction region is expected to exclude 80 per cent of the flux from the PSF. The corrected flux is consistent with the AGN fluxes obtained from the XMM-Newton spectra.

The fact that the Chandra spectrum of the nucleus requires a thermal component shows that there is a significant contribution from the galaxy also in the nuclear region. The temperature of this component is consistent with that found in the fits to the spectra from the galaxy plus jet (cf. Tables 2 and 4). The total $0.5-2 \mathrm{keV}$ flux from the emission components associated with the galaxy and the extended jet in the Chandra spectra is $8.6 \times 10^{-14} \mathrm{erg} \mathrm{cm}^{-2} \mathrm{~s}^{-1}\left(3.7 \times 10^{-14} \mathrm{erg} \mathrm{cm}^{-2} \mathrm{~s}^{-1}\right.$ and $4.9 \times 10^{-14} \mathrm{erg} \mathrm{cm}^{-2} \mathrm{~s}^{-1}$ from the nuclear region and boxes, respectively, see Tables 4 and 2). This is somewhat lower than the $2.3 \times 10^{-13} \mathrm{erg} \mathrm{cm}^{-2} \mathrm{~s}^{-1}$ found in all four XMM-Newton observations (Table 3), which is reasonable considering that the XMMNewton spectra also include the centre of the nucleus (which was excluded for the Chandra spectrum of the jet plus galaxy squared regions) as well as the galactic contribution outside the squared regions (see Fig. 1).

\section{Discussion}

In this Section we discuss the results considering several aspects raised from the imaging and spectral analysis. We start by discussing the star formation activity of the galaxy and its expected contribution to the X-ray emission in Section 4.1 and then discuss the properties of the jets and the origin of the extended Xray emission from the galaxy in Section 4.2. We finally discuss the nature of the nucleus in Section 4.3 and compare with previous results in the literature in Sect. 4.4.

\subsection{Star formation}

Since part of the X-ray emission studied in this work is of galactic origin, we start by exploring the star formation activity of the galaxy. The Star Formation Rate (SFR) of the galaxy can be constrained from the FIR luminosity, which was calculated from the fluxes at 60 and $100 \mu \mathrm{m}$ (Ranalli et al. 2003 and references therein). The FIR fluxes are $0.9 \mathrm{Jy}$ at $60 \mu \mathrm{m}$ (Neugebauer et al. 1984) and $1.58 \mathrm{Jy}$ at $100 \mu \mathrm{m}$ (reported in NED as a private communication by Knapp), which correspond to a FIR luminosity of $2 \times 10^{42} \mathrm{erg} \mathrm{s}^{-1}$ and a SFR of $0.09 \mathrm{M}_{\odot} \mathrm{yr}^{-1}$. However, this SFR is an upper limit since the FIR luminosity is dominated by the AGN (Tang et al. 2009; Asmus et al. 2014; Fernández-Ontiveros et al. 2019). We also computed an upper limit to the specific SFR (sSFR) $]^{7}$ to have a measurement of the efficiency of star formation processes. Given the stellar mass of the galaxy of $10^{11} \mathrm{M}_{\odot}$ (Fernández-Ontiveros et al. 2011), the sSFR is extremely low, $9 \times 10^{-4} \mathrm{Gyr}^{-1}$. Thus, the star formation is a rather inefficient process in NGC 1052. For comparison, in the sample of X-ray selected AGN of Rovilos et al. (2012), the lowest redshift subsample $(z<1.12)$ has sSFRs spanning a broad range of values down to $0.1 \mathrm{Gyr}^{-1}$ for the lowest luminosity sources with $\mathrm{L}_{2-10 \mathrm{keV}} \sim 4 \times 10^{41} \mathrm{erg} \mathrm{s}^{-1}$. Although the X-ray luminosity of NGC 1052 is within the low end of this sample, its sSFR is well outside. In fact, the sSFR of NGC 1052 classifies it as a 'red and dead' galaxy (Fontana et al. 2009), as already noted in Fernández-Ontiveros et al. (2011). These galaxies have rather inefficient star formation, typically with sSFR $<10^{-3} \mathrm{Gyr}^{-1}$, characterised by long time-scales.

The X-ray luminosity is correlated with the FIR luminosity in highly star forming galaxies, while AGN and LINER powered by AGN do not follow the starburst correlation due to their excess X-ray emission (Ranalli et al. 2003). From the spectral fitting, the estimated $2-10 \mathrm{keV}$ AGN luminosity is $\sim 3-4 \times 10^{41} \mathrm{erg} \mathrm{s}^{-1}$, Table 3), significantly higher than expected for a starburst source. Indeed, from the FIR luminosity of $2 \times 10^{42} \mathrm{erg} \mathrm{s}^{-1}$, the upper limit on the hard X-ray luminosity expected from star formation activity is $\mathrm{L}_{2-10 \mathrm{keV}}=$ $3.8 \times 10^{38} \mathrm{erg} \mathrm{s}^{-1}$ (from the relation in Ranalli et al. 2003). The jet plus galaxy component of the best-fit model to the XMMNewton spectra has a luminosity of $4.5 \times 10^{39} \mathrm{erg} \mathrm{s}^{-1}$ between 2 and $10 \mathrm{keV}$ (see Table 3), which is much higher due to contributions different from pure star formation.

In the soft X-ray band between 0.5 and $2 \mathrm{keV}$, the upper limit on the luminosity expected from pure star formation activity is $6.3 \times 10^{38} \mathrm{erg} \mathrm{s}^{-1}$ (Ranalli et al. 2003), while the luminosity of the thermal component in the same band from our fits to the $X M M-N e w t o n$ spectra is $\sim 2.7-3 \times 10^{39} \mathrm{erg} \mathrm{s}^{-1}$ (the thermal component contributes $\sim 30 \%$ to the jet plus galaxy emission between 0.5 and $2 \mathrm{keV}$ reported in Table 3). This shows that processes other than pure star formation make a dominant contribution to the thermal soft X-ray emission from the galaxy, as discussed below.

\subsection{Emission from the extended jet and the galaxy}

We have used the Chandra observation to explore the X-ray morphology and spectra of the extended emission in NGC 1052, including a comparison with the radio jets. VLA $1.4 \mathrm{GHz}$ (Fig. 2 of this paper) and MERLIN images (Fig. 3 of Kadler et al. 2004a) show a pair of hotspots for both the east and west lobes, which may be due to multiple jet ejection activity as in episodic radio galaxies. While this is more common in luminous sources (Nandi et al. 2019), extended radio lobes on kpc scales are not common in LINER, with only a few examples in the literature (Kozieł-Wierzbowska et al. 2012). The double structure seen at $1.4 \mathrm{GHz}$ in NGC 1052 needs higher resolution data to be con-

\footnotetext{
7 The specific star formation rate is the ratio between the SFR and the stellar mass the galaxy
} 
Table 3: Results of fits to the XMM-Newton spectra between 0.5 and $10 \mathrm{keV}$. The models are: PexJGAL: tbabs*(zpcfabs*pow+pexmon+mekal+pow). The last two mekal+pow components in the model represent the extended emission from the jet and galaxy and have parameters fixed to the model found for the jet and galaxy region from Chandra data (see Table 2 and text for details). PexiJGAL: tbabs*(zpcfabs*absori*pow+pexmon+mekal+pow). $F_{\text {AGN, } 0.5-10}$ is the sum between the primary radiation and the reflected component. All fluxes and luminosities are unabsorbed. $\mathrm{N}_{\mathrm{H} \text {,high }}$ and $\mathrm{N}_{\mathrm{H} \text {,low }}$ are the column densities of the zpcfabs and absori models, respectively, and $\xi$ is ionisation parameter of absori (defined as $L / n R^{2}$ where $L$ is the luminosity, $n$ the number density and $R$ the distance to the ionising source). The luminosities are derived from the fluxes at a distance from NGC1052 of $19.1 \mathrm{Mpc}$ (luminosity distance from NED). Refl is the reflection fraction, derived as the ratio between the fluxes of the reflected radiation to the total AGN radiation between 2 and $10 \mathrm{keV}$.

\begin{tabular}{|c|c|c|c|c|c|}
\hline PexJGAL Parameter & Unit & 0093630101 & 0306230101 & 0553300301 & 0553300401 \\
\hline$N_{\mathrm{H}}$ & {$\left[10^{22} \mathrm{~cm}^{-2}\right]$} & $10.4_{-1.7}^{+2.0}$ & $7.5_{-0.4}^{+0.5}$ & $7.8_{-0.5}^{+0.5}$ & $8.4 \pm 0.4$ \\
\hline Cov-frac & & $0.85_{-0.02}^{+0.02}$ & $0.88_{-0.01}^{+0.01}$ & $0.92_{-0.01}^{+0.01}$ & $0.94_{-0.01}^{+0.01}$ \\
\hline$\Gamma$ & & $<1.2$ & $<1.2$ & $1.3_{-0.1}^{+0.1}$ & $1.4_{-0.1}^{+0.1}$ \\
\hline$\chi^{2} /$ d.o.f. & & $168.0 / 118$ & $432.5 / 309$ & $409.5 / 298$ & $426.7 / 317$ \\
\hline PexiJGAL Parameter & & & & & \\
\hline$N_{\mathrm{H} \text {,high }}$ & {$\left[10^{22} \mathrm{~cm}^{-2}\right]$} & $25_{-6}^{+6}$ & $10_{-1}^{+1}$ & $8.7_{-0.7}^{+0.7}$ & $9.1 \pm 0.5$ \\
\hline Cov-frac & & $0.87_{-0.05}^{+0.05}$ & $0.85_{-0.02}^{+0.02}$ & $0.92_{-0.02}^{+0.01}$ & $0.92_{-0.01}^{+0.01}$ \\
\hline$\Gamma$ & & $1.82_{-0.30}^{+0.30}$ & $1.34_{-0.09}^{+0.09}$ & $1.45_{-0.09}^{+0.09}$ & $1.53_{-0.08}^{+0.08}$ \\
\hline$N_{\mathrm{H}, \text { low }}$ & {$\left[10^{22} \mathrm{~cm}^{-2}\right]$} & $2.5_{-0.8}^{+0.9}$ & $1.0_{-0.2}^{+0.3}$ & $0.6_{-0.2}^{+0.3}$ & $0.92_{-0.28}^{+0.30}$ \\
\hline$\xi$ & {$\left[\mathrm{erg} \mathrm{cm} \mathrm{s}{ }^{-1}\right]$} & $<2.1$ & $<0.4$ & $<0.2$ & $<1.9$ \\
\hline$\chi^{2} /$ d.o.f. & & $128.3 / 116$ & $385.1 / 307$ & $370.7 / 296$ & $389.2 / 315$ \\
\hline$F_{\mathrm{AGN}, 0.5-10}$ & {$\left[10^{-11} \mathrm{erg} \mathrm{s}^{-1} \mathrm{~cm}^{-2}\right]$} & $1.6 \pm 0.7$ & $0.91 \pm 0.06$ & $1.14 \pm 0.08$ & $1.26 \pm 0.08$ \\
\hline$L_{\mathrm{AGN}, 0.5-10}$ & {$\left[10^{41} \mathrm{erg} \mathrm{s}^{-1}\right]$} & $7 \pm 3$ & $3.8 \pm 0.2$ & $4.6 \pm 0.3$ & $5.2 \pm 0.3$ \\
\hline$F_{\text {jgal }, 0.5-10}$ & {$\left[10^{-13} \mathrm{erg} \mathrm{s}^{-1} \mathrm{~cm}^{-2}\right]$} & $3.30 \pm 0.10$ & $3.40 \pm 0.08$ & $3.50 \pm 0.16$ & $3.40 \pm 0.23$ \\
\hline$L_{\text {jgal }, 0.5-10}$ & {$\left[10^{40} \mathrm{erg} \mathrm{s}^{-1}\right]$} & $1.36 \pm 0.04$ & $1.40 \pm 0.04$ & $1.44 \pm 0.06$ & $1.40 \pm 0.09$ \\
\hline $\operatorname{Refl}$ & & $0.03 \pm 0.02$ & $0.06 \pm 0.01$ & $0.05 \pm 0.01$ & $0.050 \pm 0.007$ \\
\hline$F_{\mathrm{AGN}, 2-10}$ & {$\left[10^{-11} \mathrm{erg} \mathrm{s}^{-1} \mathrm{~cm}^{-2}\right]$} & $1.03 \pm 0.20$ & $0.70 \pm 0.03$ & $0.84 \pm 0.04$ & $0.90 \pm 0.02$ \\
\hline$L_{\mathrm{AGN}, 2-10}$ & {$\left[10^{41} \mathrm{erg} \mathrm{s}^{-1}\right]$} & $4.2 \pm 0.6$ & $2.9 \pm 0.1$ & $3.4 \pm 0.2$ & $3.74 \pm 0.08$ \\
\hline$F_{\text {jgal,2-10 }}$ & {$\left[10^{-13} \mathrm{erg} \mathrm{s}^{-1} \mathrm{~cm}^{-2}\right]$} & $1.1 \pm 0.05$ & $1.10 \pm 0.02$ & $1.1 \pm 0.05$ & $1.10 \pm 0.08$ \\
\hline$L_{\text {jgal,2-10 }}$ & {$\left[10^{39} \mathrm{erg} \mathrm{s}^{-1}\right]$} & $4.5 \pm 0.2$ & $4.5 \pm 0.1$ & $4.5 \pm 0.2$ & $4.5 \pm 0.3$ \\
\hline$F_{\mathrm{AGN}, 0.5-2}$ & {$\left[10^{-12} \mathrm{erg} \mathrm{s}^{-1} \mathrm{~cm}^{-2}\right]$} & $6.4 \pm 4.3$ & $2.1 \pm 0.4$ & $3.0 \pm 0.5$ & $3.6 \pm 0.5$ \\
\hline$L_{\mathrm{AGN}, 0.5-2}$ & {$\left[10^{41} \mathrm{erg} \mathrm{s}^{-1}\right]$} & $2.6 \pm 1.8$ & $0.9 \pm 0.2$ & $1.2 \pm 0.2$ & $1.5 \pm 0.2$ \\
\hline$F_{\text {jgal }, 0.5-2}$ & {$\left[10^{-13} \mathrm{erg} \mathrm{s}^{-1} \mathrm{~cm}^{-2}\right]$} & $2.2 \pm 0.1$ & $2.3 \pm 0.1$ & $2.4 \pm 0.1$ & $2.3 \pm 0.2$ \\
\hline$L_{\text {jgal }, 0.5-2}$ & {$\left[10^{39} \mathrm{erg} \mathrm{s}^{-1}\right]$} & $9.1 \pm 0.4$ & $9.5 \pm 0.4$ & $9.9 \pm 0.4$ & $9.5 \pm 0.4$ \\
\hline
\end{tabular}

firmed, but the current images make this source stand out as one of few candidates for episodic jet activity in LINER. We also note that the radio morphology of the lobes is asymmetric, with the east lobe showing a bent structure towards the south.

From the X-ray images, we find that there is soft, extended emission along the same direction as the radio structure. An Xray hotspot located at the outer edge of the eastern radio lobe is clearly seen below $2 \mathrm{keV}$. Its position indicates emission at the impact point between the jet and the environment. Similarly, there is some X-ray emission along the south-west edge of the western radio lobe. Some X-ray radiation is also seen below $1 \mathrm{keV}$ at a position coincident with the inner radio hotspot on the eastern side, but a deeper X-ray observation would be needed to confirm a possible association with the radio hotspot.

Fig. 2 shows that there is also extended emission from the galaxy that does not exactly overlap with the radio jets. Diffuse emission distributed over large scales is seen between 0.5 and $1 \mathrm{keV}$, while it is more compact between 1 and $2 \mathrm{keV}$. Moreover, we note that only the nucleus is detected above $2 \mathrm{keV}$. The different spatial scales seen between $0.5-1 \mathrm{keV}$ and $1-2 \mathrm{keV}$ suggest two ionising sources of different nature. A compact highenergy source ionises the gas emitting between 1 and $2 \mathrm{keV}$, while an extended and lower energy source ionises the gas seen between 0.5 and $1 \mathrm{keV}$. The first ionising source can be identified as the central nucleus, while the second one is instead 
Table 4: Results from fits to the Chandra spectrum of the nucleus between 0.5 and $10 \mathrm{keV}$. The models are: PexTH: tbabs (zpcfabs*pexmon + mekal); PexiTH: tbabs (zpcfabs*absori *pexmon + mekal). The photon index of the power law has not been allowed to vary below 1.1. All fluxes and luminosities are unabsorbed. The flux of the AGN component has been corrected by a factor of 5, considering that the central (excluded) region contains 80 per cent of the PSF. The errors on AGN fluxes are the average errors of the reflected and direct component.

\begin{tabular}{|c|c|c|c|}
\hline Parameter & Unit & PexTH & PexiTH \\
\hline$N_{\mathrm{H}, \text { high }}$ & {$\left[10^{22} \mathrm{~cm}^{-2}\right]$} & $12_{-3}^{+4}$ & $24_{-11}^{+30}$ \\
\hline Cov-frac & & $0.79 \pm 0.06$ & $0.6 \pm 0.1$ \\
\hline$\Gamma$ & & $<1.5$ & $1.13_{-0.03}^{+0.24}$ \\
\hline$N_{\mathrm{H}, \text { low }}$ & {$\left[10^{22} \mathrm{~cm}^{-2}\right]$} & - & $7_{-4}^{+5}$ \\
\hline$\xi$ & {$\left[\mathrm{erg} \mathrm{cm} \mathrm{s}{ }^{-1}\right]$} & - & $746_{-470}^{+4220}$ \\
\hline$T$ & {$[\mathrm{keV}]$} & $0.62_{-0.05}^{+0.05}$ & $0.71_{-0.06}^{+0.07}$ \\
\hline$\chi^{2} /$ d.o.f. & & $90.2 / 69$ & $74.8 / 67$ \\
\hline$F_{\mathrm{AGN}, 0.5-10}$ & {$\left[10^{-12} \mathrm{erg} \mathrm{cm}^{2} \mathrm{~s}^{-1}\right]$} & - & $10 \pm 6$ \\
\hline$L_{\mathrm{AGN}, 0.5-10}$ & {$\left[10^{41} \mathrm{erg} \mathrm{s}^{-1}\right]$} & - & $4 \pm 2$ \\
\hline$F_{\text {th, } 0.5-10}$ & {$\left[10^{-14} \mathrm{erg} \mathrm{cm}^{2} \mathrm{~s}^{-1}\right]$} & - & $3.8 \pm 0.4$ \\
\hline$L_{\mathrm{th}, 0.5-10}$ & {$\left[10^{39} \mathrm{erg} \mathrm{s}^{-1}\right]$} & - & $1.5 \pm 0.2$ \\
\hline $\operatorname{Refl}$ & & - & $0.05 \pm 0.05$ \\
\hline$F_{\mathrm{AGN}, 2-10}$ & {$\left[10^{-12} \mathrm{erg} \mathrm{cm}^{2} \mathrm{~s}^{-1}\right]$} & - & $8 \pm 5$ \\
\hline$L_{\mathrm{AGN}, 2-10}$ & {$\left[10^{41} \mathrm{erg} \mathrm{s}^{-1}\right]$} & - & $3.2 \pm 2.1$ \\
\hline$F_{\mathrm{th}, 2-10}$ & {$\left[10^{-15} \mathrm{erg} \mathrm{cm}^{2} \mathrm{~s}^{-1}\right]$} & - & $1.4 \pm 0.3$ \\
\hline$L_{\mathrm{th}, 2-10}$ & {$\left[10^{37} \mathrm{erg} \mathrm{s}^{-1}\right]-$} & & $5.8 \pm 1.2$ \\
\hline$F_{\mathrm{AGN}, 0.5-2}$ & {$\left[10^{-12} \mathrm{erg} \mathrm{cm}^{2} \mathrm{~s}^{-1}\right]$} & - & $1.7 \pm 0.6$ \\
\hline$L_{\mathrm{AGN}, 0.5-2}$ & {$\left[10^{40} \mathrm{erg} \mathrm{s}^{-1}\right]$} & - & $7 \pm 2$ \\
\hline$F_{\text {th }, 0.5-2}$ & {$\left[10^{-14} \mathrm{erg} \mathrm{cm}^{2} \mathrm{~s}^{-1}\right]$} & - & $3.7 \pm 0.5$ \\
\hline$L_{\mathrm{th}, 0.5-2}$ & {$\left[10^{39} \mathrm{erg} \mathrm{s}^{-1}\right]$} & - & $1.5 \pm 0.2$ \\
\hline
\end{tabular}

distributed over larger scales consistent with the scales of the ISM. This ionising radiation may come from discrete sources unresolved in the X-rays. Evidence for discrete sources in the central $1 \mathrm{kpc}$ region has been found in high-resolution NIR images (Fernández-Ontiveros et al. 2011). These sources, identified as star forming clusters, could be the reminiscence of a minor merger event reported in van Gorkom et al. (1986), connected with recent $(<1 \mathrm{Gyr})$ star formation activity. However, it is unlikely that this limited star-formation activity is sufficient to ionise the extended X-ray emission.

We fitted Chandra spectra of the regions containing emission from the extended jet and the galaxy using a phenomenological model composed of a thermal component and a power law. The thermal model represents a hot $(T \sim 0.6 \mathrm{keV})$ optically thin plasma. The temperature is consistent with shocks between the jet and the ISM, as well as emission from highly star forming regions. A similar temperature was reported in Kadler et al. (2003) and Kadler et al. (2004a), who pointed out its consistency with emission from jet-triggered shocks. The spatial distribution of emission in the Chandra images supports this scenario, as discussed above. Jet shocks convert a few percent of the kinetic energy of the jet into X-ray emission. From Kadler et al. (2003), the kinetic power of the jet is $\sim 5 \times 10^{41} \mathrm{erg} \mathrm{s}^{-1}$. The luminosity of the thermal component in the fits to the spectra of the extended emission is $\sim 8.6 \times 10^{38} \mathrm{erg} \mathrm{s}^{-1}$ (Table 2), which corresponds to $<0.2$ per cent of the jet kinetic energy, lower than the upper limits obtained in the previous Chandra observation $(<2.5$ per cent, Kadler et al. 2003). Our estimate is also an upper limit since there is most likely some contribution to the thermal emission from gas not associated with the jet.

The power-law component in our spectral fit has a less clear interpretation. Emission from the extended jet is a likely candidate given that $\mathrm{X}$-ray emission is observed along the direction of the radio lobes. However, emission from diffuse gas and weak point sources, such as X-ray binaries, may also contribute to this spectral component. If the jet contributes significantly to the power law, the steep photon index in the fit indicates that the origin may be synchrotron emission. The limited count statistic does not allow us to disentangle the possible jet emission from the environment in the spectral fitting.

Finally, we note that the XMM-Newton spectra may show evidence for emission from the galaxy in addition to the two components discussed above. Our analysis of the last XMM-Newton spectrum from 2009 (which has the best statistics) indicates a small contribution from an additional thermal component with a temperature of $1.3 \pm 0.2 \mathrm{keV}$, which might be associated with a diffuse background or discrete background sources. Such a contribution is plausible since the $\mathrm{X}$-ray image between 1 and $2 \mathrm{keV}$ 
in Fig. 1 show two background sources located south of the nucleus outside the jet plus galaxy regions, as well as some extended emission.

\subsection{The core}

We have analysed five spectra of the core of NGC 1052 (four from XMM-Newton and one from Chandra), which probe the properties of the source on time-scales from months to years. The best fitting model includes an absorbed primary power-law, contributions from the extended jets and the galaxy in the soft $\mathrm{X}$ ray band, as well as a narrow iron line at $6.4 \mathrm{keV}$ and associated Compton reflection from distant material.

The results on each of these components are discussed below. The spectral components describing the emission associated with the extended jets and galaxy in the XMM-Newton spectra were determined from the fits to the Chandra spectra of the extended emission discussed above. We allowed only the normalisation of this extended emission to vary in the fits to the $X M M-N e w t o n$ spectra, finding that it accounts for about half of the observed flux in the $0.5-2 \mathrm{keV}$ band.

\subsubsection{Primary continuum}

We find that the fluxes of the power-law components in the five observations are mostly consistent within the uncertainties, with the exception of the second XMM-Newton observation, which shows evidence for a significantly lower flux $(\sim 30 \%$ lower than in the fourth observation). Previous studies of the source have also found significant flux variability (Hernández-García et al. 2013; Connolly et al. 2016; Osorio-Clavijo et al. 2020). Compared to these works, we have somewhat larger uncertainties on the fluxes, which is due to the more complex model adopted. This will be discussed in more detail in Sect. 4.4.

We found that the primary power law makes up $\sim 95$ per cent of the unabsorbed flux above $2 \mathrm{keV}$ (Table 3), showing that accretion onto the SMBH dominates the emission at these energies. Terashima et al. (2002) discussed the possibility that galactic emission components may contribute to the spectra of LINER above $2 \mathrm{keV}$, producing a power law and/or thermal radiation at high energies. These contributions, if relevant, would complicate the spectral analysis. However, the Chandra images of NGC 1052 show extended emission primarily in the soft-Xray band. Both the spectral analysis and the images thus show that the bulk of the radiation above $2 \mathrm{keV}$ originates from the nucleus itself.

The photon indices from the XMM-Newton fits are in the range $1.34 \pm 0.09$ to $1.8 \pm 0.3$ (Table 3). These values are lower than in standard AGN, which have photon indices typically in the range between 1.8 and 2.2 (Yang et al. 2015). There are several possible explanations for the low photon index. One possibility is that emission from jets contribute to the spectrum. This may be due to a residual contribution from the extended jets or, more plausibly, a compact jet in the innermost regions. Indeed, there is evidence for an unresolved jet located in the nucleus of NGC1052 (Baek et al. 2019). This is in line with the fact that compact radio cores, which are common in LINER ( $\mathrm{Ho}$ 2008; Baldi et al. 2019), may be indicative of unresolved jets (Markoff et al. 2005, 2008). The jet scenario is also supported by the harder-when-brighter spectral variability reported in Swift/XRT observations of this source (Connolly et al. 2016). This kind of variability is commonly associated with synchroton self-Compton emission in blazars, with the increase in lu- minosity being connected to a hardening of the electron population (e.g., Ghisellini et al. 2009). This scenario has also been discussed as an explanation for the harder-when-brighter behaviour in the LLAGN NGC 7213 (Emmanoulopoulos et al. 2012). By analogy with the jet scenario, the same kind of spectral variability would be expected due to Comptonisation of synchrotron seed photons in a corona, as discussed for LLAGN in Connolly et al. (2016). In this case, the synchrotron emission may originate from the corona itself or the ADAF. Finally, an alternative possibility is that the flat X-ray continuum is due to thermal radiation from the ADAF, as argued in Guainazzi et al. (2000). These authors analysed previous observations of NGC 1052 from BebboSAX, finding that a flat power law fits the spectrum equally well as a thermal Bremsstrahlung model with a temperature of $\sim 150 \mathrm{keV}$.

\subsubsection{Absorption and reflection}

We have investigated deviations from the power-law radiation considering material around the central engine. This material can have the effect to both absorb soft X-ray photons and reprocess the higher energy radiation to produce fluorescent lines and Compton reflection. Our best-fit model comprises absorption from both a partially covering cold medium and a warm absorber. While the first one is most likely associated with cold material in the torus, the latter may be explained as material along the line of sight in the galaxy or as outflows. Recent optical spectroscopy of NGC 1052 show evidence of possible outflows, preferentially seen in [O I], in agreement with the second scenario (Cazzoli et al. 2018). However, our X-ray analysis shows an ionisation degree consistent with the neutral state.

The column densities of the clumpy and homogeneous absorbers are well determined from the spectral fitting (i.e. their uncertainties do not overlap with each other) and they are not degenerate with the other main continuum parameters like the photon index. We can conclude that the primary radiation is convolved by cold absorption in non-homogeneous material of high density $\left(\sim 9 \times 10^{22} \mathrm{~cm}^{-2}\right)$ and covering fraction of $\sim 80-90 \%$ (all parameter constraints rule out $100 \%$ coverage) and in a low density $\left(\sim 1 \times 10^{22} \mathrm{~cm}^{-2}\right)$ homogeneous gas. The column densities of the two absorbers are roughly constant across the observations with good spectral quality, with significantly different values found only in the two spectra with the lowest count statistics. We note that some previous studies have reported variations in the absorbing column (e.g., Hernández-García et al. 2013 and Osorio-Clavijo et al. 2020). As discussed in section 4.4., these discrepancies are due to differences in the models used in the different studies.

The iron $K_{\alpha}$ line at $6.4 \mathrm{keV}$ is clearly detected in all spectra. The line is well modelled by a narrow gaussian profile, which means that reflection occurs in material distant from the central engine, most likely the torus. The equivalent width measurements of 130-180 keV (see Table A.1, together with the column density estimates for the absorbers, suggest that the source is not heavily absorbed. Analysing the spectra considering iron line and Compton reflection in a self consistent way, we found that the reflection comprises 3-6\% of the unabsorbed AGN flux. The photon index of the primary radiation becomes slightly steeper when adding Compton reflection because the fit takes into account separately the (harder) reflection contribution.

We tested for the presence of relativistic reflection from the accretion disk using both the laor model to represent the iron $K_{\alpha}$ line distorted by relativistic effects, and relxill to represent the full reflection spectrum. The relativistic contribution 
does not introduce any fit improvement with respect to reflection in material distant from the SMBH. In our analysis, the constraint on the inner radius on the first XMM-Newton spectrum is $r_{\text {in }}<370 r_{\mathrm{g}}$ which does not support relativistic effects. This is expected in the ADAF scenario, where the standard disk is truncated far from the SMBH. The result is also consistent with previous studies of NGC 1052 and LINER surveys (Osorio-Clavijo et al.|2020; Hernández-García et al. 2014; González-Martín et al. 2009b), where non-relativistic reflection was used to fit the data. Our constraint on the inner radius is also larger than the $<45 r_{\mathrm{g}}$ obtained in the analysis of Suzaku data by Brenneman et al. (2009), who reported a broad line with an $\mathrm{EW}$ of $185 \mathrm{eV}$. We consider several scenarios to explain this in Section 4.4

\subsubsection{The ADAF nucleus}

To understand the accretion mode of the nucleus in NGC 1052 we computed the Eddington ratio as $\log \left(L_{\text {bol }} / L_{\text {edd }}\right)$, where $L_{\text {bol }}$ is the bolometric luminosity and $L_{\text {edd }}$ is the Eddington luminosity. Given the BH mass of $1.5 \times 10^{8} \mathrm{M}_{\odot}$ (Woo \& Urry 2002), the Eddigton luminosity is $1.8 \times 10^{46} \mathrm{erg} \mathrm{s}^{-1}$. The bolometric luminosity estimated from the integration of the Spectral Energy Distribution (SED) is $\sim 7 \times 10^{42} \mathrm{erg} \mathrm{s}^{-1}$ (Fernández-Ontiveros et al. 2019), which gives an Eddington ratio of -3.4, consistent with accretion in a radiatively inefficient mode. In this case, the optically thick, geometrically thin accretion disk is expected to be truncated and replaced by an ADAF at the centre (Ouataert et al. 1999).

Similar targets classified as LLAGN (and with Eddington ratios similar to NGC 1052) have a transition radius between the standard disk and the ADAF around 100 Schwarzschild radi 8 , as discussed in Yuan \& Narayan (2004). However, modelling of the SED of NGC 1052 with an ADAF-scenario has yielded mixed results. While Yuan et al. (2009) finds that the source is well described by an ADAF-dominated model, the ADAF+jet model of Yu et al. (2011) significantly over-predicts the UV flux, which is instead better described by a standard disk extending to the innermost stable circular orbit. More recently, Reb et al. (2018) analysed the sub-arcsec SED and found that the standard disk is truncated at a radius $>26 r_{\mathrm{g}}$. The scenario of a truncated disk is also supported by our analysis, which shows that the X-ray spectra lack signs of relativistic reflection.

\subsection{Comparison with previous works}

Here we discuss our findings regarding the nucleus and surrounding regions in NGC 1052 in relation to previous studies of the X-ray properties of the source. Our analysis of the extended emission includes the first comparison between images from the 2005 Chandra observation and VLA observations. The X-ray images show diffuse emission extended along the radio lobes, as well as hotspots associated with the edges of the lobes. This confirms previous results obtained by Kadler et al. (2004a) with a much shorter Chandra observation. While the diffuse X-ray emission has a similar extent at the radio lobes, the detailed structures do not correlate well, which indicates that the X-ray and radio emission trace different parts of the particle population in the extended jets. Indeed, differences between X-rays and radio are commonly seen in jets, including the nearby well-observed cases of Cen A and M87 (Kraft et al. 2002; Perlman \& Wilson 2005). In addition to offsets between

\footnotetext{
8 The Schwarzschild radius is $2 \times r_{g}$
}

$\mathrm{X}$-ray and radio hotspots in the jets of both these sources, the Cen A jet is also narrower in X-rays than in radio (Kraft et al. 2002).

We characterised the spectrum of the circum-nuclear emission, disentangling it from the nucleus. The area selected for this study includes the X-ray emission from the radio lobes as well as part of the galaxy, but excludes the central core. An estimate of the circum-nuclear spectrum from the same data was recently reported in Osorio-Clavijo et al. (2020), based on an annular extraction region beween $3^{\prime \prime}$ and $25^{\prime \prime}$. They find a thermal component consistent with our results, but a significantly flatter power law $(0.7 \pm 0.2$ compared to our value of $2.3 \pm 0.2)$. This discrepancy is likely due to differences in the extraction region. Since $10 \%$ of the emission from a point source is outside 3 arcsec at $8.5 \mathrm{keV}$ 9), it is plausible that the harder photon index reported by Osorio-Clavijo et al. (2020) is affected by a small contribution from the bright and hard nucleus. Our extraction region excludes the central $6^{\prime \prime}$ radius, which ensures that there is no emission from the nucleus contributing to the spectrum. The different circum-nuclear spectrum likely also explains the slightly lower AGN fluxes in Osorio-Clavijo et al. (2020), considering that part of the nucleus may be included in the circum-nuclear model. From our analysis, the AGN power law of the spectra with good statistics are also somewhat harder (all spectra except for the first, lowest quality spectrum from XMM-Newton), again probably due to the new characterisation of the circum-nuclear spectrum.

The overall model for the nucleus adopted in this work contains an absorber composed by partially covering material and a warm absorber, with physical parameters broadly consistent with previous works, considering the uncertainties. We thus confirm previous findings from Osorio-Clavijo et al. (2020) and Brenneman et al. (2009), which show that non-homogeneous cold absorption in combination with a lower density absorbing material fully covering the source fit the data better than a simple cold uniform absorber. The model used to fit the $X M M$ Newton spectra also requires a small contribution from reflection in material distant from the central SMBH. Although the overall model provides an acceptable fit to the broad-band spectra, we found indications for an additional thermal component that improves the fit in the soft X-rays. We argue that this might be the result of a small contribution from background radiation outside the two jet+galaxy regions.

Our analysis reveals weak evidence for variability in the best-fit spectral parameters (see Table 3). The first XMMNewton observation and the Chandra observation show higher values of the column densities of the absorbers, but these two observations also have the lowest spectral quality. There is also marginal evidence for variability in $\Gamma$. Previous studies of NGC 1052 have revealed significant variations in hardness ratios using observations from both XMM-Newton (Hernández-García et al. 2013) and Swift (Connolly et al. 2016). The analysis of Hernández-García et al. (2013) mainly attributed this to changes in the absorbing column, while Connolly et al. (2016) favoured a varying photon index. In comparison to these works, we have considered a more comprehensive physical scenario with a larger number of free parameters. From this analysis we cannot attribute the spectral variability to a specific parameter or component of the model.

Using a model that is more similar to ours, Osorio-Clavijo et al. (2020) find small variability in $\Gamma$ and the column density of the clumpy absorber between the three

\footnotetext{
9 http://cxc.harvard.edu/proposer/POG/html/chap4.html
} 
best XMM-Newton observations (the ones from 2006-Jan, 2009-Jan, and 2009-Aug). The tighter constraints on these parameters from Osorio-Clavijo et al. (2020) arise from the fact that some of the other model parameters were kept constant or tied across the observations, whereas we have allowed all the parameters in Table 3 to vary independently. We thus conclude that the spectral variability between these three observations is small enough that it does not result in significant differences between the best-fit parameters when allowing all of them to vary.

Our analysis does not reveal a significant relativistic iron line as was reported for the previous Suzaku data (Brenneman et al. 2009). The relativistic line in the Suzaku spectrum had an EW of $185 \mathrm{eV}$ and gave a constraint on the inner disk radius of $<45$ $r_{\mathrm{g}}$. We consider different scenarios to understand this difference with respect to our results. We start by noting that it cannot be explained by differences in the spectral quality because the best XMM-Newton spectra have 35000 counts in the X-ray band, while the Suzaku data have 39000 total counts from 0.5 to $9 \mathrm{keV}$. However, we note that the first XMM-Newton spectrum has a hint of a red wing of the line, although it does not clearly stand out from the background noise (see Fig. 4 , panel a). The possibility of a relativistic contribution to the line in the same spectrum was noted in Ros et al. (2007), who also discussed a possible connection with a jet ejection that would have occurred in 2001, as found in radio data, right before the XMM-Newton observation. In the case of the Suzaku data, which does not show any Compton reflection associated with the relativistic line, it was also proposed that the line emission may originate in the base of the jet (Brenneman et al. 2009). Line emission from the base of the jet is not expected to arise together with Compton reflection and could in principle be variable on time scales of years, as discussed in Ros et al. (2007). However, we cannot verify this because of the limited spectral quality of the XMM-Newton spectrum of 2001 (it has only 4000 counts).

The detection of relativistic line emission in the Suzaku spectrum of 2007 could alternatively be explained, at least in part, as due to a different way to model the continuum in Brenneman et al. (2009). The continuum model for the Suzaku spectrum was determined from fits that ignore the spectral band beween $3 \mathrm{keV}$ and $7 \mathrm{keV}$ to exclude the iron line region. The 3 to $7 \mathrm{keV}$ band was then reintroduced when fitting the relativistic line, which may lead to an underestimate of the continuum. Indeed, it is extremely rare to find relativistic lines extended down to $3 \mathrm{keV}$; even in luminous AGN the most extreme cases extend down to $4 \mathrm{keV}$ (see for instance Nandra et al. (2007) or Risaliti et al. (2013) for a more recent analysis). Our continuum model for the XMM-Newton spectra differs from the one used for the Suzaku spectra in that it includes the emission components inferred from the Chandra spectrum of the jet and the galaxy. The inclusion of these soft X-ray components affects the other parameters of the fit (including the absorbers and the powerlaw), thus modifying the continuum model also at high energies. Consequently, the more detailed continuum characterisation may affect the conclusions regarding the presence of a relativistic Fe line.

Taken at face value, the relativistic iron line found in Suzaku but not detected in the XMM-Newton observations, can finally be interpreted as the genuine result of some variability in the innermost regions of the accretion disc. We checked if different pictures besides the jet ejection event can explain variability in the relativistic line, considering in particular instabilities in the accretion disk (e.g. McHardy et al. 2006 and Merloni et al. 2003). We thus compare the fluxes measured in the Suzaku ob- servation of July 2007 with the closest XMM-Newton observations, obtained in January 2006 and January 2009. The latter have fluxes between 2 and $10 \mathrm{keV}$ of $\sim 7.0 \pm 0.3$ and $\sim 8.4 \pm 0.4$ $10^{-12} \mathrm{erg} \mathrm{cm}^{-2} \mathrm{~s}^{-1}$, respectively, marginally lower than the measurement from Suzaku of $9 \pm 1 \times 10^{-12} \mathrm{erg} \mathrm{cm}^{-2} \mathrm{~s}^{-1}$ reported in Brenneman et al. (2009). We conclude that there is no significant continuum flux variability in support of accretion disk transitions.

\section{Conclusions}

We have studied one Chandra observation and four XMMNewton observations of NGC 1052 in order to explore its innermost structure along the scales of the radio jets and to determine the nature of the emission. Our main conclusions are the following:

- The Chandra images reveal diffuse emission below $1 \mathrm{keV}$ extended along the radio lobes. However, the diffuse X-ray emission does not correlate well with the radio contours, suggesting that the X-ray and radio emission trace different parts of the particle distribution of the extended jets. In addition, there is a faint hotspot at the border of the eastern radio lobe and also some emission along the southern edge of the western lobe. This can be explained as emission from shocks between the extended jets and the environment.

- The Chandra spectrum extracted from the regions containing emission from the extended jets and galaxy can be described by a thermal component and a power law. While the main contribution to the thermal component are the X-ray hotspots coincident with the radio lobes, the power law likely represents the emission from the jets, with a possible contribution from other weak sources in the galaxy. A comparison with the FIR luminosity shows that star formation is expected to make a very small contribution to the $\mathrm{X}$-ray emission.

- From the images, we see that the main emission above $2 \mathrm{keV}$ originates from the nucleus. The spectral analysis shows that the continuum from the nucleus is well modelled by a hard power law $(\Gamma \sim 1.4)$. This can be interpreted as the emission from a hot ADAF, a compact jet, or Comptonisation in a corona, where the seed photons in the latter case may be synchrotron photons from the ADAF or the corona itself.

- The continuum spectrum is absorbed by a complex system comprising a high density $\left(N_{\mathrm{H}, \text { high }} \gtrsim 9 \times 10^{22} \mathrm{~cm}^{-2}\right)$ patchy torus (covering fraction $\sim 90$ per cent) and a diffuse absorbing structure with a lower density $\left(N_{\mathrm{H} \text {,low }} \lesssim 2 \times 10^{22} \mathrm{~cm}^{-2}\right)$, likely associated with diffuse gas along the line of sight.

- A narrow iron line at $6.4 \mathrm{keV}$ is significantly detected in all spectra of the nucleus, providing clear evidence of accretion onto the central SMBH. The associated Compton reflection from distant material accounts for 3-6 \% of the total AGN flux between 2 and $10 \mathrm{keV}$.

- The spectra lack a relativistic line at $6.4 \mathrm{keV}$. This means that the inner edge of the accretion disk is too far away from the central SMBH for relativistic effects to be detected. The spectral analysis constrains the inner radius to $<370 r_{\mathrm{g}}$, considering a continuum composed of a power law and Compton reflection. This is in agreement with radiatively inefficient accretion in the form of an ADAF hosted in a truncated accretion disk.

Acknowledgements. We thank the referee for the constructive comments, which have greatly improved the paper. JL and SF thank the Knut \& Alice Wallenberg Foundation and the Swedish National Space Board. SN acknowledges support by the Science \& Engineering Research Board, a statutory body of Department of Science and Technology (DST), Government of India (FILE NO. 
PDF/2018/002833). This research has made use of data obtained from the Chan dra Data Archive and software provided by the Chandra X-ray Center (CXC) in the application packages CIAO and CHIPS. This research has made use of the NASA/IPAC Extragalactic Database (NED), which is operated by the Jet Propulsion Laboratory, California Institute of Technology, under contract with the National Aeronautics and Space Administration.

\section{References}

Alexander, D. M. \& Hickox, R. C. 2012, New A Rev., 56, 93

Alonso-Herrero, A., Pérez-González, P. G., Rieke, G. H., et al. 2008, ApJ, 677, 127

Armus, L., Heckman, T. M., \& Miley, G. K. 1990, ApJ, 364, 471

Asmus, D., Hönig, S. F., Gandhi, P., Smette, A., \& Duschl, W. J. 2014, MNRAS 439,1648

Baczko, A. K., Schulz, R., Kadler, M., et al. 2019, A\&A, 623, A27

Baczko, A. K., Schulz, R., Kadler, M., et al. 2016, A\&A, 593, A47

Baek, J., Chung, A., Schawinski, K., et al. 2019, MNRAS, 488, 4317

Baldi, R. D., Capetti, A., \& Giovannini, G. 2019, MNRAS, 482, 2294

Balmaverde, B. \& Capetti, A. 2015, A\&A, 581, A76

Brenneman, L. W., Weaver, K. A., Kadler, M., et al. 2009, ApJ, 698, 528

Cazzoli, S., Márquez, I., Masegosa, J., et al. 2018, MNRAS, 480, 1106

Celotti, A., Ghisellini, G., \& Chiaberge, M. 2001, MNRAS, 321, L1

Collins, J. A. \& Rand, R. J. 2001, in Astronomical Society of the Pacific Conference Series, Vol. 240, Gas and Galaxy Evolution, ed. J. E. Hibbard, M. Rupen, \& J. H. van Gorkom, 392

Connolly, S. D., McHardy, I. M., Skipper, C. J., \& Emmanoulopoulos, D. 2016, MNRAS, 459, 3963

Dauser, T., Garcia, J., Parker, M. L., Fabian, A. C., \& Wilms, J. 2014, MNRAS 444, L100

Dopita, M. A., Ho, I. T., Dressel, L. L., et al. 2015, ApJ, 801, 42

Dopita, M. A., Koratkar, A. P., Allen, M. G., et al. 1997, ApJ, 490, 202

Emmanoulopoulos, D., Papadakis, I. E., McHardy, I. M., et al. 2012, MNRAS, 424, 1327

Evans, P. A., Beardmore, A. P., Page, K. L., et al. 2007, A\&A, 469, 379

Falocco, S., Carrera, F. J., Barcons, X., Miniutti, G., \& Corral, A. 2014, A\&A, 568, A 15

Fernández-Ontiveros, J. A., López-Gonzaga, N., Prieto, M. A., et al. 2019, MNRAS, 485, 5377

Fernández-Ontiveros, J. A., López-Sanjuan, C., Montes, M., Prieto, M. A., \& Acosta-Pulido, J. A. 2011, MNRAS, 411, L21

Fontana, A., Santini, P., Grazian, A., et al. 2009, A\&A, 501, 15

Fruscione, A., McDowell, J. C., Allen, G. E., et al. 2006, in Proc. SPIE, Vol. 6270, Society of Photo-Optical Instrumentation Engineers (SPIE) Conference Series, $62701 \mathrm{~V}$

García, J., Dauser, T., Lohfink, A., et al. 2014, ApJ, 782, 76

Ghisellini, G., Maraschi, L., \& Tavecchio, F. 2009, MNRAS, 396, L105

González-Martín, O., Díaz-González, D., Acosta-Pulido, J. A., et al. 2014, A\&A, 567, A92

González-Martín, O., Masegosa, J., Márquez, I., \& Guainazzi, M. 2009a, ApJ, 704,1570

González-Martín, O., Masegosa, J., Márquez, I., Guainazzi, M., \& JiménezBailón, E. 2009b, A\&A, 506, 1107

González-Martín, O., Masegosa, J., Márquez, I., et al. 2015, A\&A, 578, A74

Guainazzi, M. \& Antonelli, L. A. 1999, MNRAS, 304, L15

Guainazzi, M., Oosterbroek, T., Antonelli, L. A., \& Matt, G. 2000, A\&A, 364 L80

Hardcastle, M. J. \& Worrall, D. M. 1999, MNRAS, 309, 969

Heckman, T. M. 1980, A\&A, 500, 187

Hernández-García, L., González-Martín, O., Márquez, I., \& Masegosa, J. 2013, A\&A, 556, A47

Hernández-García, L., González-Martín, O., Masegosa, J., \& Márquez, I. 2014, A\&A, 569, A26

Hernández-García, L., Masegosa, J., González-Martín, O., Márquez, I., \& Perea, J. 2016, ApJ, 824, 7

Hickox, R. C., Jones, C., Forman, W. R., et al. 2009, ApJ, 696, 891

Ho, L. C. 2008, Araa, 46, 475

Jensen, J. B., Tonry, J. L., Barris, B. J., et al. 2003, ApJ, 583, 712

Kadler, M., Kerp, J., Ros, E., et al. 2004a, A\&A, 420, 467

Kadler, M., Ros, E., Kerp, J., et al. 2003, New A Rev., 47, 569

Kadler, M., Ros, E., Lobanov, A. P., Falcke, H., \& Zensus, J. A. 2004b, A\&A, 426, 481

Kozieł-Wierzbowska, D., Jamrozy, M., Zola, S., Stachowski, G., \& Kuźmicz, A. 2012, MNRAS, 422, 1546

Kraft, R. P., Forman, W. R., Jones, C., et al. 2002, ApJ, 569, 54

Laor, A. 1991, ApJ, 376, 90

Markoff, S., Nowak, M., Young, A., et al. 2008, ApJ, 681, 905

Markoff, S., Nowak, M. A., \& Wilms, J. 2005, ApJ, 635, 1203
McHardy, I. M., Koerding, E., Knigge, C., Uttley, P., \& Fender, R. P. 2006, Nature, 444, 730

Merloni, A., Heinz, S., \& di Matteo, T. 2003, MNRAS, 345, 1057

Nandi, S., Saikia, D. J., Roy, R., et al. 2019, MNRAS, 486, 5158

Nandra, K., O’Neill, P. M., George, I. M., \& Reeves, J. N. 2007, MNRAS, 382, 194

Narayan, R., Mahadevan, R., Grindlay, J. E., Popham, R. G., \& Gammie, C. 1998, ApJ, 492, 554

Neugebauer, G., Soifer, B. T., Rice, W., \& Rowan-Robinson, M. 1984, PASP, 96, 973

Osorio-Clavijo, N., González-Martín, O., Papadakis, I. E., Masegosa, J., \& Hernández-García, L. 2020, MNRAS, 491, 29

Perlman, E. S. \& Wilson, A. S. 2005, ApJ, 627, 140

Plucinsky, P. P., Bogdan, A., Marshall, H. L., \& Tice, N. W. 2018, in Society of Photo-Optical Instrumentation Engineers (SPIE) Conference Series, Vol. 10699, Proc. SPIE, 106996B

Quataert, E., Di Matteo, T., Narayan, R., \& Ho, L. C. 1999, ApJ, 525, L89

Ranalli, P., Comastri, A., \& Setti, G. 2003, A\&A, 399, 39

Reb, L., Fernández-Ontiveros, J. A., Prieto, M. A., \& Dolag, K. 2018, MNRAS, 478, L122

Risaliti, G., Harrison, F. A., Madsen, K. K., et al. 2013, Nature, 494, 449

Ros, E., Kadler, M., Kaufmann, S., et al. 2007, in Highlights of Spanish Astrophysics IV, ed. F. Figueras, J. M. Girart, M. Hernanz, \& C. Jordi, 165

Rovilos, E., Comastri, A., Gilli, R., et al. 2012, A\&A, 546, A58

Sugai, H. \& Malkan, M. A. 2000, ApJ, 529, 219

Tang, Y., Gu, Q.-S., Huang, J.-S., \& Wang, Y.-P. 2009, MNRAS, 397, 1966

Tavecchio, F., Maraschi, L., Sambruna, R. M., \& Urry, C. M. 2000, ApJL, 544, L23

Terashima, Y., Iyomoto, N., Ho, L. C., \& Ptak, A. F. 2002, ApJS, 139, 1

Tully, R. B., Courtois, H. M., Dolphin, A. E., et al. 2013, AJ, 146, 86

van Gorkom, J. H., Knapp, G. R., Raimond, E., Faber, S. M., \& Gallagher, J. S. 1986, AJ, 91,791

Woo, J.-H. \& Urry, C. M. 2002, ApJ, 581, L5

Worrall, D. M. 2009, A\&AR, 17,

Yang, Q.-X., Xie, F.-G., Yuan, F., et al. 2015, MNRAS, 447, 1692

Yu, Z., Yuan, F., \& Ho, L. C. 2011, ApJ, 726, 87

Yuan, F. \& Narayan, R. 2004, ApJ, 612, 724

Yuan, F., Yu, Z., \& Ho, L. C. 2009, ApJ, 703, 1034 

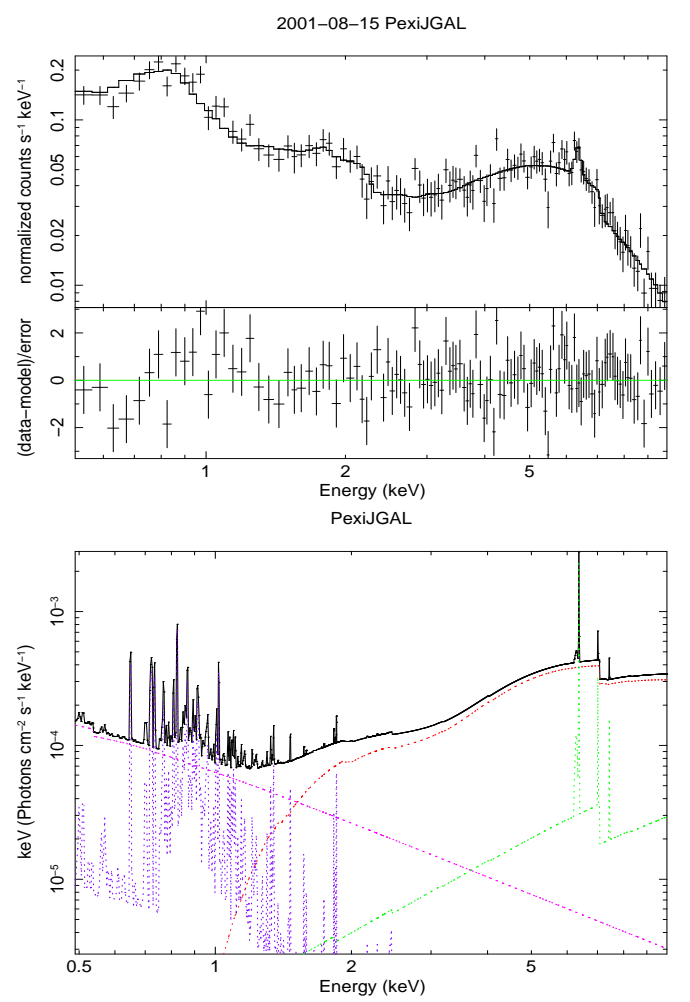

(a) Spectrum of 2001 .
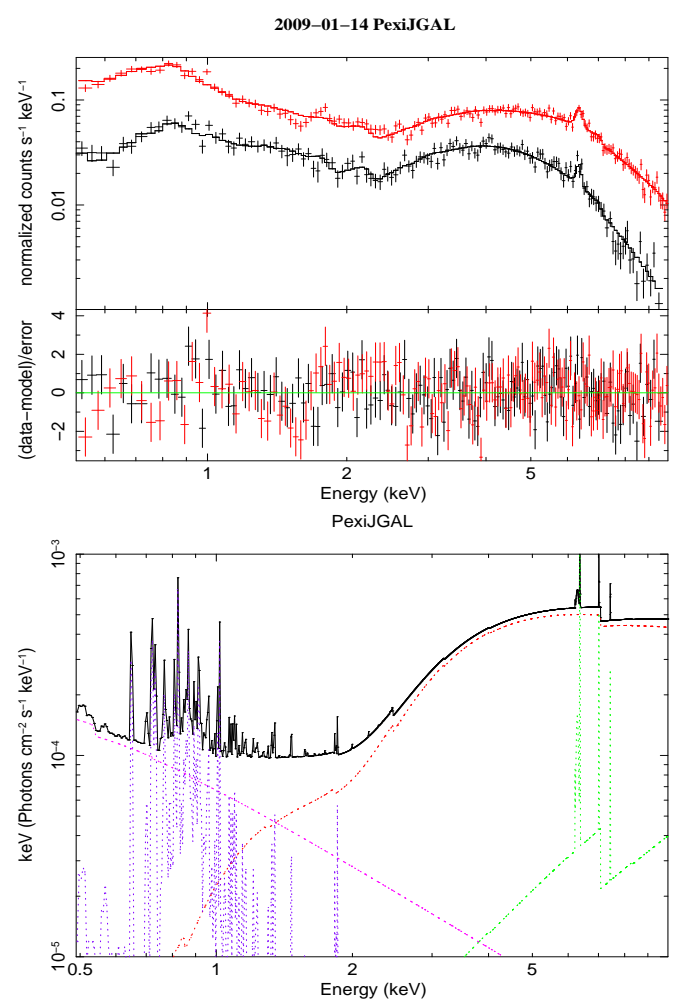

(c) Spectrum of 2009 January.
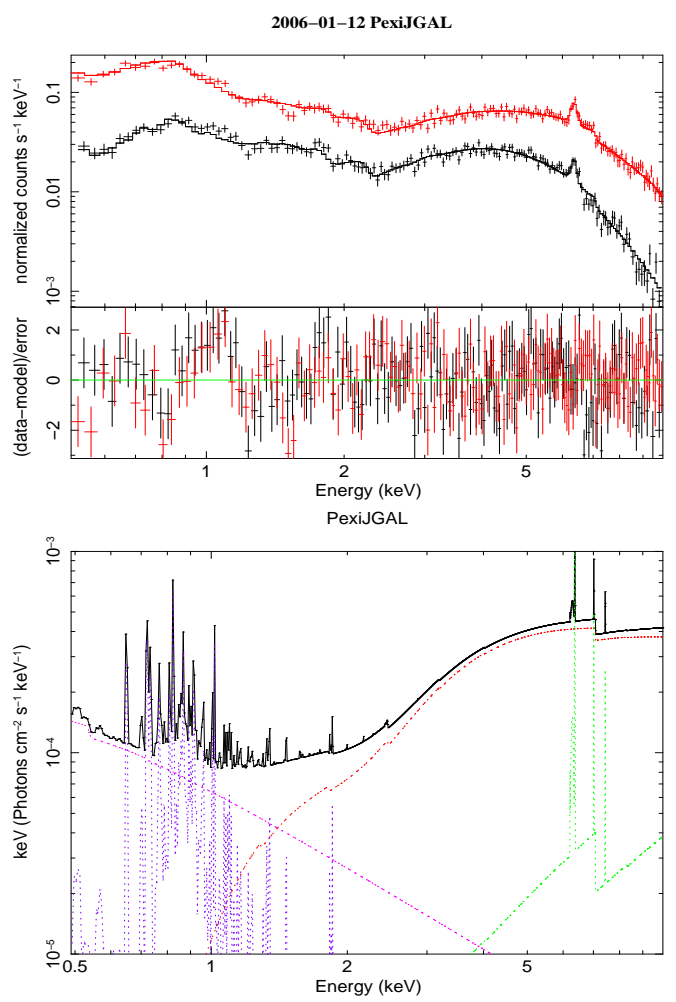

(b) Spectrum of 2006.
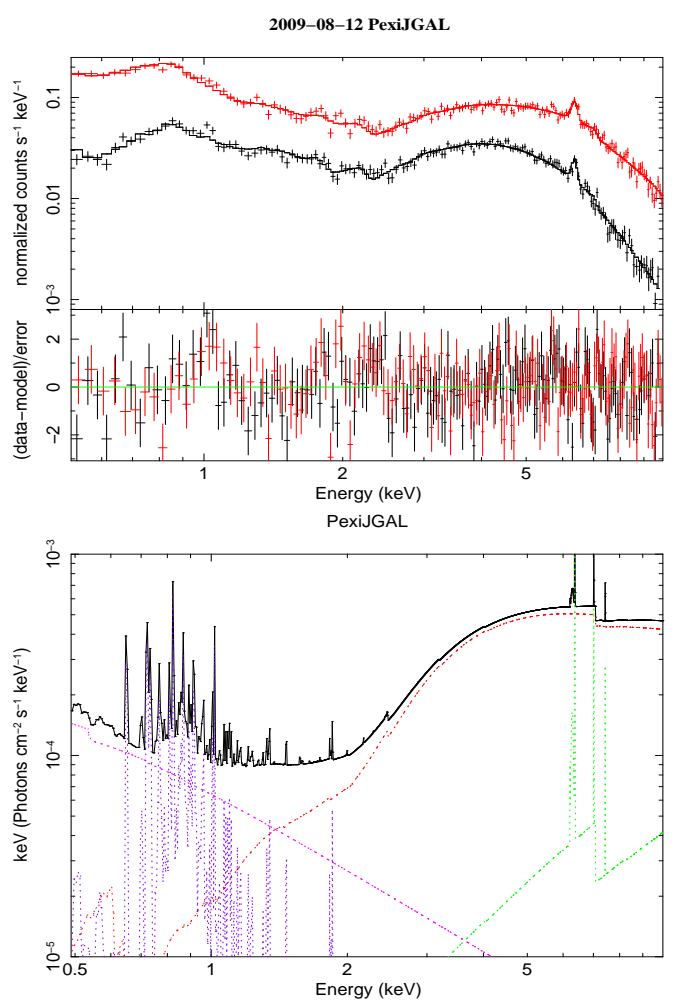

(d) Spectrum of 2009 August.

Fig. 4: XMM-Newton spectra fitted with the PexiJGAL model (black line), together with the fit residuals and the individual model components. The red line is the absorbed power law from the nucleus and the green line is the pexmon model. The blue and magenta lines are the jet plus galaxy model 

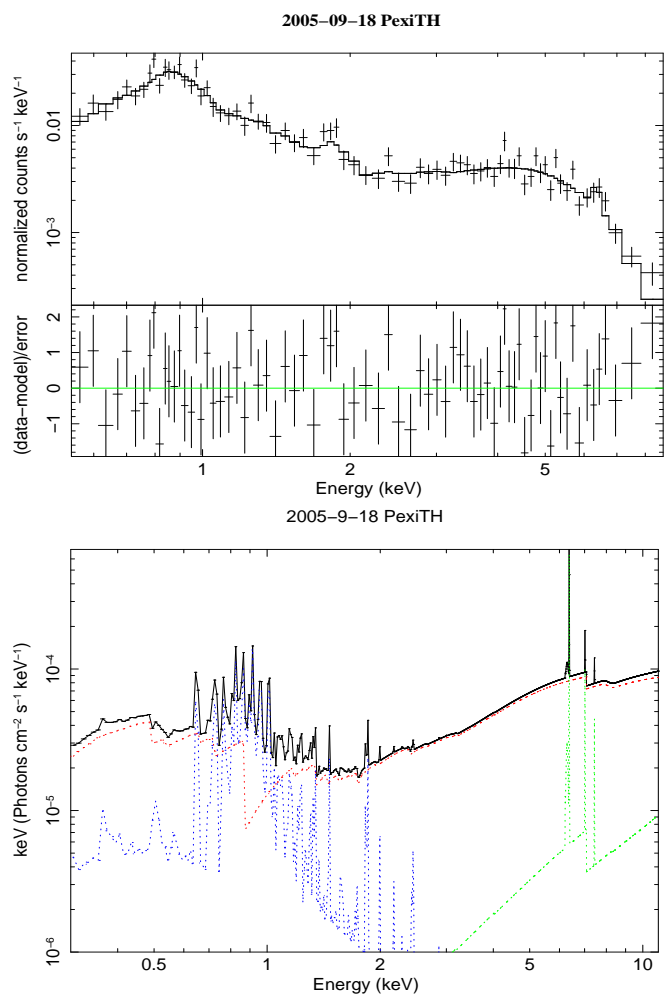

Fig. 5: The Chandra spectrum of the nucleus from 2005 fitted with the PexiTH model (black line). The model components are shown in the lower panel. The red line is the absorbed power law from the nucleus, the green line is the pexmon model, and the blue line is the mekal component from the galaxy. 


\section{Appendix A: Fits with simple continuum models}

This section reports fits parameters, fluxes and luminosities estimated from the XMM-Newton nuclear spectra fitting the spectra with simple models which ignore Compton reflection. The fit results are presented in Table A.1. The first two entires in the table are the P-JGAL and PG-JGAL models introduced in section 3 . The third model was obtained by modifying PG-JGAL model introduced in Sect. 3, to take into account extra absorption as we did for the fits reported in Sect. 3 . For this, we used a warm absorber (absori), giving the total model tbabs (zpcfabs*absori*pow + zgauss + mekal + pow). The model provides an acceptable fit to all four spectra and the main spectral parameters are broadly consistent with the ones reported in Sect. 3 . 
Table A.1: Results of fits to the XMM-Newton spectra between 0.5 and $10 \mathrm{keV}$. The models are PJGAL: tbabs*(zpcfabs*pow+mekal+pow). PG-JGAL: tbabs*(zpcfabs*pow+GAUSS+mekal+pow). PGI-JGAL: tbabs*(zpcfabs*absori*pow+GAUSS+mekal+pow). The last two mekal+POW components in all three models represent the extended emission from the jet and galaxy (see Table 2 and text for details). All fluxes and luminosities are unabsorbed. $\mathrm{N}_{\mathrm{H}, \text { high }}$ and $\mathrm{N}_{\mathrm{H} \text {,low }}$ are the column densities of the zpcfabs and absori models, respectively, and $\xi$ is ionisation parameter of absori. EW is the equivalent width of the narrow iron line.

\begin{tabular}{|c|c|c|c|c|c|c|}
\hline \multirow[b]{2}{*}{ Model } & \multirow[b]{2}{*}{ Parameter } & \multirow[b]{2}{*}{ Unit } & \multicolumn{4}{|c|}{ OBSID } \\
\hline & & & 0093630101 & 0306230101 & 0553300301 & 0553300401 \\
\hline \multirow[t]{2}{*}{ P-JGAL } & & & & & & \\
\hline & $\chi^{2} /$ d.o.f. & & $231.0 / 119$ & $571.1 / 310$ & 499.0/299 & $563.0 / 318$ \\
\hline \multirow[t]{6}{*}{ PG-JGAL } & & & & & & \\
\hline & $N_{\mathrm{H}, \text { high }}$ & {$\left[10^{22} \mathrm{~cm}^{-2}\right]$} & $16_{-2}^{+2}$ & $7.4_{-0.5}^{+0.5}$ & $7.7_{-0.5}^{+0.5}$ & $8.2_{-0.4}^{+0.4}$ \\
\hline & Cov-frac & & $0.90_{-0.02}^{+0.02}$ & $0.88_{-0.01}^{+0.01}$ & $0.920_{-0.008}^{+0.008}$ & $0.930_{-0.005}^{+0.005}$ \\
\hline & $\Gamma$ & & $1.51_{-0.09}^{+0.09}$ & $1.05_{-0.07}^{+0.07}$ & $1.24_{-0.06}^{+0.06}$ & $1.32_{-0.06}^{+0.06}$ \\
\hline & EW & {$[\mathrm{eV}]$} & $180_{-65}^{+65}$ & $183_{-25}^{+25}$ & $150_{-26}^{+26}$ & $152_{-22}^{+22}$ \\
\hline & $\chi^{2} /$ d.o.f. & & $211.8 / 118$ & $430.2 / 309$ & $406.2 / 298$ & $430.7 / 317$ \\
\hline \multirow[t]{8}{*}{ PGI-JGAL } & & & & & & \\
\hline & $N_{\mathrm{H}, \text { high }}$ & {$\left[10^{22} \mathrm{~cm}^{-2}\right]$} & $25_{-5}^{+6}$ & $10_{-1}^{+1}$ & $8.6_{-0.6}^{+0.7}$ & $9.0_{-0.5}^{+0.6}$ \\
\hline & Cov-frac & & $0.86_{-0.06}^{+0.04}$ & $0.84_{-0.02}^{+0.02}$ & $0.91_{-0.01}^{+0.01}$ & $0.92_{-0.01}^{+0.01}$ \\
\hline & $\Gamma$ & & $1.72_{-0.31}^{+0.33}$ & $1.27_{-0.02}^{+0.02}$ & $1.38_{-0.08}^{+0.09}$ & $1.46_{-0.07}^{+0.07}$ \\
\hline & $N_{\mathrm{H}, \text { low }}$ & {$\left[10^{22} \mathrm{~cm}^{-2}\right]$} & $2.34_{-0.79}^{+0.87}$ & $0.99_{-0.21}^{+0.27}$ & $0.59_{-0.22}^{+0.35}$ & $0.90_{-0.29}^{+0.27}$ \\
\hline & $\xi$ & {$\left[\operatorname{erg~cm~s}{ }^{-1}\right]$} & $<2.5$ & $<0.6$ & $<0.2$ & $<2.5$ \\
\hline & EW & {$[\mathrm{eV}]$} & $136_{-61}^{+61}$ & $169_{-24}^{+24}$ & $146_{-23}^{+23}$ & $148_{-22}^{+22}$ \\
\hline & $\chi^{2} /$ d.o.f. & & $129.7 / 116$ & $385.7 / 307$ & $370.4 / 296$ & $394.5 / 315$ \\
\hline
\end{tabular}

